Draft VERSION OCTOBER 8, 2018

Preprint typeset using $\mathrm{LATE}_{\mathrm{E}} \mathrm{X}$ style emulateapj v. 5/2/11

\title{
A NEW MULTI-DIMENSIONAL GENERAL RELATIVISTIC NEUTRINO HYDRODYNAMICS CODE FOR CORE-COLLAPSE SUPERNOVAE II. RELATIVISTIC EXPLOSION MODELS OF CORE-COLLAPSE SUPERNOVAE
}

\author{
Bernhard Müller, Hans-Thomas Janka, Andreas Marek \\ Max-Planck-Institut für Astrophysik, Karl-Schwarzschild-Str. 1, D-85748 Garching, Germany; bjmuellr@mpa-garching.mpg.de, \\ thj@mpa-garching.mpg.de \\ Draft version October 8, 2018
}

\begin{abstract}
We present the first two-dimensional general relativistic (GR) simulations of stellar core collapse and explosion with the CoCoNuT hydrodynamics code in combination with the VERTEX solver for energy-dependent, three-flavor neutrino transport, using the extended conformal flatness condition for approximating the spacetime metric and a ray-by-ray-plus ansatz to tackle the multi-dimensionality of the transport. For both of the investigated 11.2 and $15 M_{\odot}$ progenitors we obtain successful, though seemingly marginal, neutrino-driven supernova explosions. This outcome and the time evolution of the models basically agree with results previously obtained with the Prometheus hydro solver including an approximative treatment of relativistic effects by a modified Newtonian potential. However, GR models exhibit subtle differences in the neutrinospheric conditions compared to Newtonian and pseudo-Newtonian simulations. These differences lead to significantly higher luminosities and mean energies of the radiated electron neutrinos and antineutrinos and therefore to larger energy-deposition rates and heating efficiencies in the gain layer with favorable consequences for strong nonradial mass motions and ultimately for an explosion. Moreover, energy transfer to the stellar medium around the neutrinospheres through nucleon recoil in scattering reactions of heavy-lepton neutrinos also enhances the mentioned effects. Together with previous pseudo-Newtonian models the presented relativistic calculations suggest that the treatment of gravity and energy-exchanging neutrino interactions can make differences of even $50-100 \%$ in some quantities and is likely to contribute to a finally successful explosion mechanism on no minor level than hydrodynamical differences between different dimensions.

Subject headings: supernovae: general-neutrinos-radiative transfer-hydrodynamics - relativity
\end{abstract}

\section{INTRODUCTION}

More than 40 years after the first attempts by Colgate \& White (1966), the quest for the supernova explosion mechanism is still ongoing. Since the solution now seems (once again) within reach as several groups have come up with successful explosion models (Marek \& Janka 2009; Suwa et al. 2010; Takiwaki et al. 2012; Bruenn et al. 2009; Burrows et al. 2006, 2007a), the demand for accurate predictions of the neutrino and gravitational wave signals and of the nucleosynthetic yields becomes more urgent and naturally requires increased levels of sophistication in numerical core-collapse supernova simulations.

Currently, there is a broad consensus that multidimensional hydrodynamical instabilities are one of the pivotal elements of the supernova problem. It has long been recognized that convection in the hot-bubble region serves to increase the efficiency of neutrino heating behind the shock (Herant et al. 1992, 1994; Burrows et al. 1995; Janka \& Müller 1996; Müller \& Janka 1997), and that another more recently discovered large-scale instability, the standing accretion-shock instability ("SASI", Blondin et al. 2003; Blondin \& Mezzacappa 2006; Foglizzo et al. 2006; Ohnishi et al. 2006; Foglizzo et al. 2007; Scheck et al. 2008; Iwakami et al. 2008, 2009; Fernández \& Thompson 2009; Fernández 2010) has a similar beneficial effect. Both instabilities help to keep the accreted material in the gain region for a longer time before it is advected deeper into the cooling region and onto the neutron star surface (Buras et al. 2006a; Murphy \& Burrows 2008). If the advection time-scale $\tau_{\mathrm{adv}}$ through the gain region (sometimes also termed "residence time" of the matter in the gain region) is increased sufficiently and becomes comparable to or larger than the heating time-scale $\tau_{\text {heat }}$ required to unbind the material between gain radius and shock, a runaway situation occurs, in which neutrino heating leads to shock expansion, which in turn lengthens the residence time, thus again increasing the neutrino heating efficiency (Janka \& Keil 1998; Thompson 2000; Janka et al. 2001; Buras et al. 2006a; Thompson et al. 2005; (Murphy \& Burrows 2008).

However, whether an explosion can actually be brought underway in this fashion in the most sophisticated supernova models with detailed neutrino transport has not yet been finally and unambiguously established on the basis of state-of-the-art neutrino hydrodynamics simulations in 2D. Using their Vertex-Prometheus code, which employs a variable Eddington factor technique to solve the neutrino moment equations and the "ray-by-ray-plus" approach to cope with multidimensional transport, the Garching group has found explosions by the SASI-aided neutrino-driven mechanism for an $11.2 M_{\odot}$ solar mass progenitor (Buras et al. 2006a; Marek \& Janka 2009), which can be reproduced robustly for stiffer and softer nuclear equations of state, (Marek et al. in preparation) and for a $15 M_{\odot}$ progenitor with artificially imposed rotation at a rather late time $\approx 550 \mathrm{~ms}$ after bounce. In other cases, i.e. for the same $15 M_{\odot}$ progenitor without rotation and with a better ef- 
fective gravitational potential, and for more massive progenitors, no explosion was observed until 400 - $500 \mathrm{~ms}$ after bounce. In contrast to this, the Oak Ridge group, relying on a multi-group flux-limited diffusion (MGFLD) algorithm combined with the ray-by-ray-plus approach, has obtained explosions for a host of different progenitors (Bruenn et al. 2006, 2009), while the former Arizona group did not obtain neutrino-driven explosions at all with their 2D MGFLD code VuLCAN (Livne et al. 2004, 2007) but found acoustic explosions powered by sound waves created by violent dipolar oscillations of the protoneutron star (Burrows et al. 2006, 2007b). Using yet another neutrino transport scheme, the "isotropic diffusion source approximation" (Liebendörfer et al. 2009) in the ray-by-ray-approximation and without $\nu_{\mu}$ and $\nu_{\tau}$ transport, an explosion has also been reported by Suwa et al. (2010) and Takiwaki et al. (2012) for an $11.2 M_{\odot}$ and a $13 M_{\odot}$ progenitor.

Given the disparity of methods and input physics, these different results should not be overly surprising: None of the aforementioned groups follow approaches that are completely identical with respect to the treatment of neutrino transport (variable Eddington factor method vs. MGFLD vs. IDSA, inclusion/omission of non-isoenergetic scattering, velocity effects, and gravitational redshift, ray-by-ray transport vs. multi-angle transport), the neutrino processes (e.g. omission of $\mu / \tau$ neutrinos in Suwa et al. 2010 and Takiwaki et al. 2012), the hydro solver (high-resolution shock capturing schemes vs. artificial viscosity), or the treatment of gravity (effective relativistic potential vs. Newtonian approximation) and nuclear burning (network vs. flashing vs. omission of burning). A clear sensitivity of supernova simulations to Newtonian vs. GR gravity, the sophistication of the neutrino opacities, and observer corrections in the transport equations has recently been demonstrated in 1D models by Lentz et al. (2012b). Different results depending on the input physics and the approximations used by the different groups are all the more to be expected considering that some of the $2 \mathrm{D}$ models, whether exploding or non-exploding, appear to be marginal cases (see e.g. Marek \& Janka 2009) anyway. From this perspective, the viability of the neutrino-driven mechanism in $2 \mathrm{D}$ remains a controversial issue and should still be investigated further with the help of better simulations.

Considering the status of current 2D core-collapse supernova models, it is conceivable that they still miss a crucial ingredient for robust explosions. Alternatives to the standard neutrino-driven mechanism have therefore been proposed and explored, such as magnetohydrodynamically-driven explosions (see Burrows et al. 2007a and references therein), energy input by acoustic waves (Burrows et al. 2006, 2007b), or a phase transition to quark matter (Sagert et al. 2009). Moreover, 3D effects have recently been advocated as the decisive factor by Nordhaus et al. (2010) in their comparison of $1 \mathrm{D}, 2 \mathrm{D}$, and $3 \mathrm{D}$ simulations using a strongly simplified prescription for neutrino heating and cooling. Adopting the conceptual view of Burrows \& Goshy (1993) of the neutrino-driven mechanism as a critical phenomenon, they report a reduction of the so-called critical luminosity in 3D by $15 \%-25 \%$ compared to $2 \mathrm{D}$, and single out the dimensionality as the key to successful supernova explosions, discounting other factors such as general relativity and detailed neutrino microphysics as minuscule corrections. However, whether these results, obtained by means of a very rough approximation for neutrino heating and cooling, can be verified by simulations with an elaborate transport treatment is yet unclear (see Hanke et al. 2011; Takiwaki et al. 2012). Moreover, the lower estimate of Nordhaus et al. (2010) for the importance of $3 \mathrm{D}$ effects relative to $2 \mathrm{D}$ would probably not make them the single most important factor in supernova physics, at least not by far. While there is no doubt that 3D models are indispensable for better understanding the SASI (Blondin \& Mezzacappa 2007; Iwakami et al. 2008, 2009; Fernández 2010), the morphology of supernova explosions, and the kicks and spins of neutron stars (Hammer et al. 2010; Wongwathanarat et al. 2010; Rantsiou et al. 2011; Fernández 2010), our understanding of supernovae certainly does not hinge on dimensionality alone.

General relativity (GR) is undoubtedly another major factor in supernova physics due to the compactness of the neutron star $(M / R \approx 0.1 \ldots 0.2$ in geometrized units) and the occurrence of large infall $(v / c \approx$ $-0.15 \ldots-0.3)$ and outflow velocities. In spherical symmetry (1D), the gauge freedom can be exploited to take GR effects into account without sacrificing accuracy in the neutrino transport sector more readily than in 2D and 3D; gray and multi-group flux-limited diffusion schemes in 1D (Baron et al. 1989; Bruenn et al. 2001) have therefore been available since the 1980s and were followed by general relativistic Boltzmann solvers a few years ago (Yamada et al. 1999; Liebendörfer et al. 2001, 2004). Comparisons with the Newtonian case in 1D (Bruenn et al. 2001) clearly showed the importance of GR effects, particularly at late times during the accretion phase, where Bruenn et al. (2001) find a much stronger recession of the shock ( $50 \%$ smaller radius), higher luminosities, in particular for electron neutrinos and antineutrinos (by $\approx 40 \%$ ) and higher mean neutrino energies (by $\approx 15 \%$ ) in the GR case. This strong sensitivity to GR effects was recently confirmed with up-to-date neutrino microphysics by Lentz et al. (2012b). Considering the magnitude of relativistic effects, it is obvious that they ought to be properly considered in multi-dimensional supernova models as well.

Unfortunately, until recently the only viable option to include GR effects in multi-dimensional neutrino hydrodynamics simulations while retaining the framework of Newtonian hydrodynamics has been the use of modified gravitational or "effective" potentials based on the Tolman-Oppenheimer-Volkov equation of stellar structure (Rampp \& Janka 2002; Marek et al. 2006; Müller et al. 2008). This "pseudo-Newtonian" approach is easy to implement, yields very satisfactory results in 1D (Liebendörfer et al. 2005; Marek et al. 2006; Müller et al. 2010), and certainly provides a rough firstorder approximation for GR effects in multi-dimensional supernova models. As a complementary approach starting from multi-dimensional general relativistic hydrodynamics simulations, there have been efforts to address certain aspects of core-collapse supernovae with the help of heavily simplified neutrino treatments such as a "deleptonization scheme" (Liebendörfer 2005) or a trapping scheme, but the applicability of such methods is naturally limited, e.g. to the collapse and bounce phase, 
(Ott et al. 2007a; Dimmelmeier et al. 2007a, 2008), or fast black hole formation (Ott et al. 2011).

In this paper, we pursue a considerably more ambitious course and present the first 2D core-collapse supernova models combining general relativistic hydrodynamics and a sophisticated energy-dependent neutrino transport scheme. Using the VERTEX-CoCoNuT code with a ray-by-ray-plus treatment of multi-flavor, multi-energy 2D neutrino transport as documented in Müller et al. (2010, henceforth Paper I), we have conducted simulations of two progenitor models with $11.2 M_{\odot}$ and $15 M_{\odot}$ beyond the onset of the explosion. These simulations are complemented by three non-exploding runs of the $15 M_{\odot}$ progenitor, viz. one with a pseudo-Newtonian (effective potential) treatment of gravity, one with purely Newtonian gravity, and one with a simplified set of neutrino interaction rates. The major purpose of these simulations can be summarized as follows,

1. to demonstrate the feasibility of full-scale multidimensional general relativistic supernova simulations,

2. to evaluate the role of general relativistic effects in the explosion mechanism and the quality of the pseudo-Newtonian approach,

3. to gauge the sensitivity of the heating conditions to the neutrino physics input,

4. to closely reproduce and verify pseudo-Newtonian simulations with the PrometheUs-VERTEX code (Buras et al. 2006a; Marek \& Janka 2009) using a different hydrodynamics solver, thus eliminating reservations about the reliability of the numerics in existing $2 \mathrm{D}$ supernova models.

Naturally, the impact of GR on the gravitational wave (and also on the neutrino) signal from core-collapse supernovae is also a question of paramount importance. However, this issue cannot be discussed within the scope of the present paper with its focus on the dynamics of the post-bounce evolution, and will be the subject of a follow-up publication.

To address the aforementioned issues, we have structured our paper in the following manner: We review the numerical scheme and outline the model setup and input physics used in this work in Sections 2 and 3 . In Section 4. we describe the shock evolution and the explosion morphology of our relativistic explosion models. In Section 5. we then address differences to the nonexploding runs and provide a more quantitative analysis of the heating conditions for our models in order to determine the impact of the GR treatment and the neutrino microphysics on the post-bounce dynamics. We summarize the main results from this analysis in Section 6 and evaluate the implications with respect to the major issues of our paper.

\section{NUMERICAL METHODS}

We perform numerical simulations with the general relativistic neutrino hydrodynamics code VERTEXCoCoNuT introduced in Paper I (Müller et al. 2010), which is a combination of the neutrino transport solver VERTEX (Rampp \& Janka 2002; Buras et al. 2006b) and the relativistic hydrodynamics code CoCoNuT (Dimmelmeier et al. 2002, 2005). CoCoNuT is a timeexplicit, directionally unsplit Eulerian Godunov-type finite-volume solver written for spherical polar coordinates and uses piecewise parabolic (PPM) reconstruction and Runge-Kutta time-stepping to achieve higherorder spatial and temporal accuracy. Our implementation of CoCoNuT employs a relativistic version of the HLLC approximative Riemann solver (Mignone \& Bodo 2005), but adaptively switches to the more diffusive HLLE solver (Einfeldt 1988) in the vicinity of strong shocks to avoid the phenomenon of odd-even-decoupling Quirk 1994). In order to reduce spurious numerical energy generation, we use an improved formulation of the energy equation described in Paper I. The metric equations are solved approximatively using the extended conformal flatness condition (xCFC, Cordero-Carrión et al. 2009), a constrained scheme with improved numerical stability properties compared to the original conformally flat approximation of Isenberg (1978). In the context of core-collapse, this approximation works extremely well as demonstrated by the excellent agreement between rotational core-collapse simulations using the CFC approximation and those relying on the full ADM formalism (Ott et al. 2007a, b; Dimmelmeier et al. 2007b).

The time-implicit neutrino transport module VERTEX integrates the moment equations for the neutrino energy and momentum density using a variable Eddington factor technique (Rampp \& Janka 2002). The higher moments of the neutrino radiation field that are required to close the system of moment equations are obtained from a simplified Boltzmann equation that is solved in conjunction with the neutrino moment equations within a fixed-point iteration. All velocity- and metric-dependent terms are fully included in the moment equations, as is energy redistribution by non-isoenergetic scattering. In 2D, we resort to the "ray-by-ray-plus" approximation (Buras et al. 2006b; Bruenn et al. 2006), assuming that the neutrino distribution function is axially symmetric around the radial direction (which implies a radial flux vector). This allows us to solve independent $1 \mathrm{D}$ transport problems along "rays" corresponding to the angular zones of the polar grid. However, the lateral advection of neutrinos and the effect of lateral neutrino pressure gradients are both included to avoid unphysical behavior in the optically thick regime (Buras et al. 2006b).

We use an up-to-date set of neutrino interaction rates in VERTEX; in addition to the "standard" set of neutrino opacities from Bruenn (1985), we take nucleon-nucleon bremsstrahlung (Hannestad \& Raffelt 1998), neutrinoneutrino pair conversion (Buras et al. 2003) and inelastic neutrino scattering off heavy nuclei (Langanke et al. 2008) into account. Furthermore, we compute electron captures on heavy nuclei using the improved rate tables of Langanke et al. (2003) instead of the FFN rates (Fuller et al. 1982), and as an alternative to the "isoenergetic" (i.e. no energy exchange with nucleons treated as vacuum particles) approximation of Bruenn (1985) we include recoil effects, high-density correlations (Burrows \& Sawver 1998, 1999), and weak magnetism corrections (Horowitz 1997) in our treatment of charged and neutral-current neutrino-nucleon interactions (see Table 2). 


\section{MODEL SETUP}

\subsection{Progenitors and Neutrino Physics}

In this paper, we consider two different non-rotating progenitors, namely the models s11.2 of Woosley et al. (2002) and s15s7b2 of Woosley \& Weaver (1995). These $11.2 M_{\odot}$ and $15 M_{\odot}$ progenitors have been chosen such as to facilitate a comparison with the pseudo-Newtonian simulations of Buras et al. (2006a) and Marek \& Janka (2009). The $15 M_{\odot}$ case is of particular interest since Marek \& Janka (2009) observed an explosion for this progenitor in a run with artificially imposed rotation and an overly strong effective gravitational potential (their model LS-rot) while the corresponding non-rotating model (LS-2D), computed with the best effective potential of Marek et al. (2006), failed to explode before the end of their high-resolution run $420 \mathrm{~ms}$ after bounce. Within the model assumptions of Marek \& Janka (2009), the progenitor s15s7b2 is therefore a marginal case, and hence ideally suited for investigating possible effects of general relativity and testing the accuracy of the effective potential approach. In addition to two relativistic models (G11 and G15) for the two progenitors computed with VERTEX-COCONuT, we therefore also consider a purely Newtonian (N15) and a pseudo-Newtonian (M15) simulation of the $15 M_{\odot}$ star of Woosley \& Weaver (1995) computed with VERTEX-PROMETHEUS.

Furthermore, we also include another relativistic calculation (S15) with a slightly simplified set of neutrino opacities to assess the importance of improved interactions rates (particularly for neutrino-nucleon processes). A summary of the input physics for these models is given in Table 1, and the differences between the full and simplified set of neutrino rates are given in detail in Table 2. Model S15 serves to illustrate the possible variations in 2D core-collapse supernova simulations that may be due the treatment of the neutrino microphysics. Since a full investigation of all the individual rates in multi-dimensional supernova simulations is not feasible for lack of computer power, we choose a "package" of opacities for model S15 that is roughly representative for the neutrino treatment used in the 1980s and 1990s and, together with model G15, spans a reasonable part of the range of sophistication of the neutrino microphysics introduced in modern multi-dimensional core-collapse simulations. While model S15 thus provides some rough indications about the influence of the neutrino rates in core-collapse supernovae, it should be borne in mind that even more radical approximations than our "simplified" set of interaction rates are used (e.g. $\mu$ - and $\tau$ - neutrinos are sometimes ignored completely, or treated by a leakage/trapping scheme), which can affect the dynamics much more seriously.

With the exception of model N15 (where 128 angular zones were used), all runs were performed on a spherical polar grid covering $180^{\circ}$ in latitude with $400 \times 64$ zones initially. We simulate the interior of each progenitor out to $10000 \mathrm{~km}$, corresponding to a mass coordinate of $1.57 M_{\odot}(\mathrm{s} 11.2)$ and $2.0 M_{\odot}(\mathrm{s} 15 \mathrm{~s} 7 \mathrm{~b} 2)$, respectively. The distribution of the radial grid varies between the individual simulation: 1 , but was chosen (and when necessary re-

\footnotetext{
1 Specifically, stability considerations require different zoning in the hydrodynamics modules CoCoNuT and Prometheus near the
}

adjusted) such that the density gradient at the surface of the proto-neutron star remains sufficiently well resolved during the simulation. With a moderate angular resolution of $2.8^{\circ}$ we have settled for an affordable compromise in the first long-time multi-dimensional simulations using the relativistic VERTEX-COCONUT code. For future calculations (relying on more efficient parallelization of the hydrodynamics and metric solvers), a higher resolution is clearly desirable, but past experience with pseudoNewtonian simulations suggests that 64 angular zones already provide a reasonable level of accuracy to study systematic differences between models with different input physics. Both for the $11.2 M_{\odot}$ and the $15 M_{\odot}$ progenitor, this resolution is in fact sufficient for good quantitative agreement with high-resolution studies in the pseudoNewtonian case as demonstrated by the similarity of our model M15 with model LS-2D of Marek \& Janka 2009. For comparisons or extrapolation, it should nonetheless be borne in mind that runs with higher angular resolution generally appear somewhat more optimistic in $2 \mathrm{D}$ (cp. Scheck 2006 for numerical tests with approximate, gray neutrino transport and Hanke et al. 2011 for recent tests with simple neutrino source terms). The reason for this empirical trend is yet to be determined, although Hanke et al. (2011) suggest that the inverse turbulent energy cascade in 2D, which may shift energy from high$\ell$ convective modes into low $\ell$ SASI modes, could provide an explanation. The effect could be connected to a reduction of the dissipation on small scales with higher resolution or to additional energy input into convective motions on the smallest availables scales. We refer the reader to their paper for a more thorough discussion of this issue.

For the neutrino transport, we chose a logarithmicallyspaced energy grid with 12 bins ranging from $0 \mathrm{MeV}$ to $380 \mathrm{MeV}$, which - though not an optimal resolution yields fairly similar dynamics compared to a finer zoning in energy space (cp. Marek \& Janka 2009 for an example with only 9 bins).

\subsection{Equation of State}

The simulations were performed using a soft version of the equation of state (EoS) of Lattimer \& Swesty (1991) with a bulk incompressibility modulus of nuclear matter of $K=180 \mathrm{MeV}$ (LS180). The value of $K$ in this EoS has raised some objections (Nordhaus et al. 2010; Dasgupta et al. 2012) because measurements point to $K=240 \mathrm{MeV}$ for symmetric nuclear matter (Shlomo et al. 2006), and because observations have recently pushed the maximum neutron star mass to at least $2 M_{\odot}$ (Demorest et al. 2010), which is incompatible with this particular EoS (see Figure 1 and cp. also Hempel et al. 2012).

However, this criticism neglects that (at least for the range of progenitors we are considering here) the crucial quantity for the development of the explosion is not the maximum neutron star mass, but rather the radius of the proto-neutron star during the reheating phase. For the baryonic neutron star masses we obtain at the end of our longest-running simulations $\left(1.35 M_{\odot}\right.$ and $1.58 M_{\odot}$ for the $11.2 M_{\odot}$ and $15 M_{\odot}$ progenitor, respectively), the LS180 EoS yields a final radius (in the cold state) of

origin of the spherical polar grid. 
Table 1

Model Setup

\begin{tabular}{ccccccc}
\hline \hline model & progenitor & $\begin{array}{c}\text { neutrino } \\
\text { opacities }\end{array}$ & $\begin{array}{c}\text { hydro } \\
\text { solver }\end{array}$ & $\begin{array}{c}\text { treatment of } \\
\text { relativity }\end{array}$ & $\begin{array}{c}\text { final post-bounce } \\
\text { time reached }^{\text {a }}\end{array}$ & $\begin{array}{c}\text { angular } \\
\text { resolution }^{\circ}\end{array}$ \\
\hline G11 & s11.2 & full set & CoCoNUT & GR hydro + xCFC & $920 \mathrm{~ms}$ & $2.8^{\circ}$ \\
G15 & s15s7b2 & full set & CoCoNuT & GR hydro + xCFC & $775 \mathrm{~ms}$ & $2.8^{\circ}$ \\
S15 & s15s7b2 & reduced set & CoCoNUT & GR hydro + xCFC & $474 \mathrm{~ms}$ & $2.8^{\circ}$ \\
M15 & s15s7b2 & full set & PROMETHEUs & Newtonian + modified potential & $517 \mathrm{~ms}$ & $2.8^{\circ}$ \\
N15 & s15s7b2 & full set & PROMETHEUs & Newtonian (purely) & $525 \mathrm{~ms}$ & $1.4^{\circ}$ \\
\hline \hline
\end{tabular}

an practice, technical reasons limit the simulation time. The five simulations discussed here all needed to be terminated because convergence of the implicit transport solver could only be ensured by inordinately small time-steps at late times. Such convergence problems occur because of extremely steep velocity gradients in spots where fast downflows strike the proto-neutron star surface.

${ }^{\mathrm{b}}$ Case A of Marek et al. (2006)

Table 2

Neutrino physics input

\begin{tabular}{|c|c|c|}
\hline process & $\begin{array}{c}\text { full rates } \\
(\mathrm{G} 11, \mathrm{G} 15, \mathrm{M} 15, \mathrm{~N} 15)\end{array}$ & $\begin{array}{c}\text { simplified rates } \\
(\mathrm{S} 15)\end{array}$ \\
\hline \multirow[t]{2}{*}{$\nu A \rightleftharpoons \nu A$} & Horowitz (1997) (ion-ion correlations) & Bruenn \& Mezzacappa (1997) \\
\hline & Langanke et al. (2008) (inelastic contribution) & Itoh et al. (2004) (ion-ion-correlations) \\
\hline$\nu e^{ \pm} \rightleftharpoons \nu e^{ \pm}$ & Mezzacappa \& Bruenn (1993) & Mezzacappa \& Bruenn (1993) \\
\hline$\nu N \rightleftharpoons \nu N$ & Burrows \& Sawver (1998) & Bruenn (1985) \\
\hline$\nu_{e} n \rightleftharpoons e^{-} p$ & Burrows \& Sawyer $(1998)^{a}$ & Bruenn (1985) \\
\hline $\bar{\nu}_{e} p \rightleftharpoons e^{+} n$ & Burrows \& Sawyer $(1998)^{a}$ & Bruenn (1985) \\
\hline$\nu_{e} A^{\prime} \rightleftharpoons e^{-} A$ & Langanke et al. (2003) & Fuller et al. (1982); Bruenn (1985) \\
\hline$\nu \bar{\nu} \rightleftharpoons e^{-} e^{+}$ & Bruenn (1985); Pons et al. (1998) & Bruenn (1985); Pons et al. (1998) \\
\hline$\nu \bar{\nu} N N \rightleftharpoons N N$ & Hannestad \& Raffelt (1998) & Hannestad \& Raffelt (1998) \\
\hline$\nu_{\mu, \tau} \bar{\nu}_{\mu, \tau} \rightleftharpoons \nu_{e} \bar{\nu}_{e}$ & Buras et al. (2003) & - \\
\hline$\left.\left(_{\nu}^{-}\right)_{\mu, \tau}(-)\right)_{e} \rightleftharpoons\left(-\frac{(-)}{\nu}\right)_{\mu, \tau}\left(-\frac{(-)}{\nu}\right)_{e}$ & Buras et al. (2003) & - \\
\hline
\end{tabular}

a Note that these reaction rates account for nucleon thermal motions, phase-space blocking, energy transfer to the nucleon associated with recoil ("non-isoenergetic" scattering), and nucleon correlations at high densities. Moreover, we include the quenching of the axial-vector coupling at high densities (Carter \& Prakash 2002), correction to the effective nucleon mass (Reddy et al. 1999), and weak magnetism effects (Horowitz 2002) in our full set of rates.

around $12 \mathrm{~km}$ (see Figure 1), which is smaller by only $0.5 \ldots 0.7 \mathrm{~km}$ than the neutron star radius predicted by the ("stiffer") LS220 EoS (which has $K=220 \mathrm{MeV}$ and yields a maximum neutron star mass larger than $\left.2 M_{\odot}\right)$. Figure 1 also shows that the deviation is of similar magnitude for hot proto-neutron stars. Moreover, a neutron star radius of $12 \mathrm{~km}$ is well compatible with observed neutron star radii; it actually agrees nicely with the best existing (observational and theoretical) estimates of neutron star radii, see e.g. the papers by Steiner et al. (2010) and Hebeler et al. (2010), a fact that, for example, disfavors the EoS of Shen et al. (1998), because the latter yields a radius of $\approx 15 \mathrm{~km}$ for a neutron star with a gravitational mass of $1.4 M_{\odot}$. The prospective differences to a simulation with LS220 become even smaller if we consider that the two neutron stars are even less massive when the explosion develops (e.g. at a baryonic mass of $1.51 M_{\odot}$ for the $15 M_{\odot}$ progenitor).

It is also important to note that the neutron stars formed in the $11.2 M_{\odot}$ and $15 M_{\odot}$ runs have baryonic masses of $1.35 M_{\odot}$ and $1.58 M_{\odot}$, but the corresponding gravitational masses are about $\approx 10 \%$ lower than these values. These masses are far below the maximum gravitational mass that can be supported by the LS180 EOS (which is about $1.85 M_{\odot}$, corresponding to a baryonic mass well beyond $2 M_{\odot}$; Figure 11).
In the neutron star mass regime we are dealing with in this paper, LS180 therefore remains a justifiable choice for the EoS. This "softer EoS" yields neutron star radii quite similar to the "stiffer" version LS220 because of relatively small differences in the pressure-density relation. It is also very similar in most other EoS properties. Sizable differences appear only close to the limiting neutron star masses supported by these equations of state.

\section{EXPLOSION DYNAMICS AND ENERGETICS}

The relativistic supernova simulations of the $11.2 M_{\odot}$ and $15 M_{\odot}$ stars discussed in this paper both yield successful explosions. In this section, we address the evolution of models G11 and G15 in a descriptive manner, focusing on the propagation of the shock, the activity of the SASI, the morphology of the explosion, and the ejecta composition. The heating conditions in our models and the crucial differences responsible for the different outcome of the $15 M_{\odot}$ models G15, M15, N15, and S15 will be analyzed in the next section.

Both the $11.2 M_{\odot}$ and the $15 M_{\odot}$ models show the development of prompt post-shock convection a few milliseconds after bounce, which then triggers early SASI activity at low amplitude for $\approx 50 \mathrm{~ms}$. This can be seen in Figure 3. where we show the normalized coefficients $a_{\ell}$ of the decomposition of the angle-dependent shock posi- 

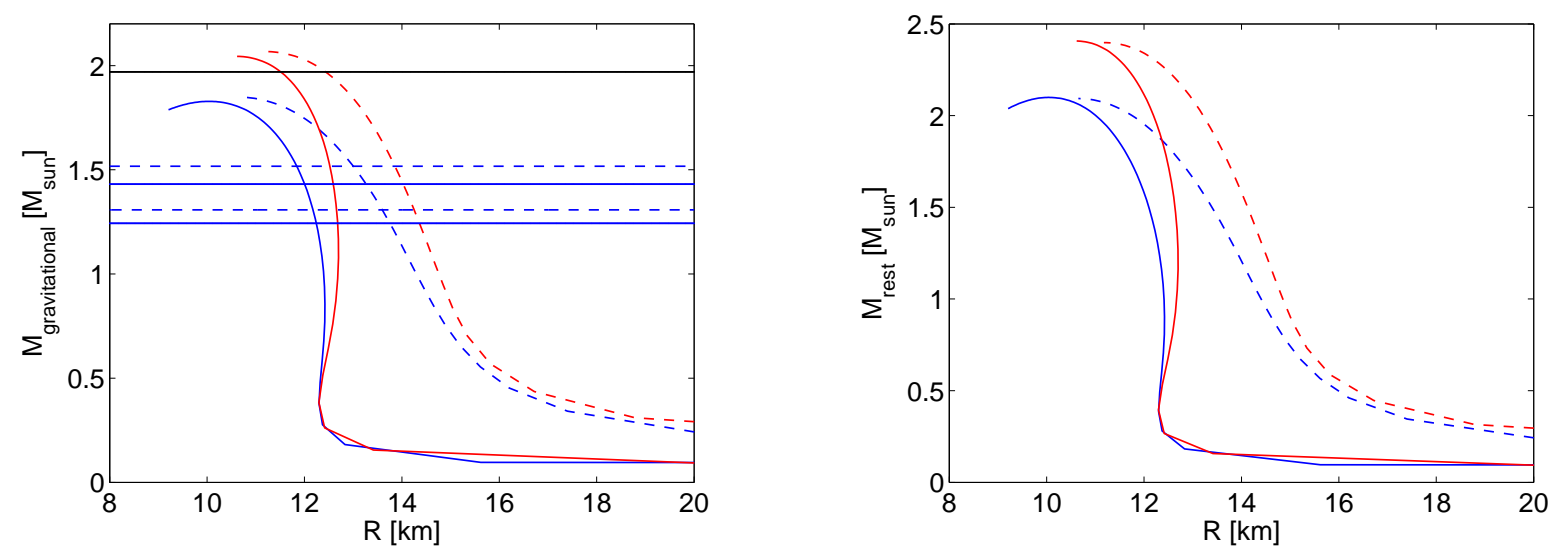

Figure 1. Mass-radius relations of the equations of state LS180 (blue) and LS220 (red) for the gravitational mass (left panel) and the baryonic mass (right panel). Solid lines display the case of cold neutron stars $(T=0)$, while curves for the case of a hot proto-neutron star with a constant entropy of $s=1.5 \mathrm{k}_{b}$ /nucleon are shown as dashed lines. The black horizontal line in the left panel corresponds to a mass of $1.97 M_{\odot}$ as measured by Demorest et al. (2010) for the pulsar J1614-2230. The gravitational masses for neutron stars with baryonic masses of $1.36 M_{\odot}$ and $1.58 M_{\odot}$ are indicated both for $T=0$ (solid blue horizontal lines) or $s=1.5 \mathrm{k}_{b} /$ nucleon (dashed blue horizontal lines) in the left panel (figures provided by A. Bauswein.)

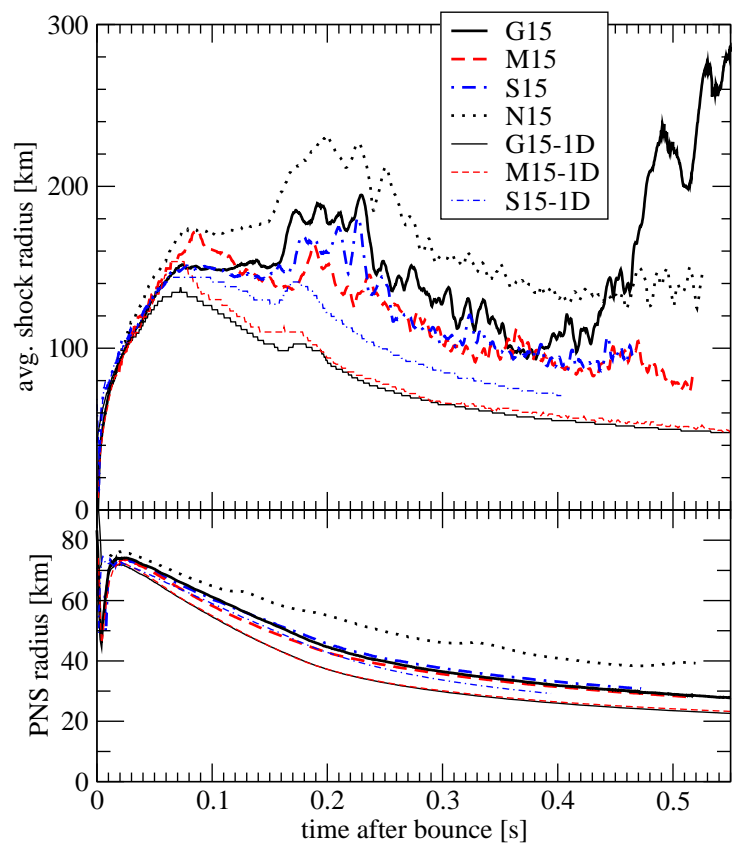

Figure 2. Average shock radius and proto-neutron star (PNS) radius (defined by a fiducial density of $10^{11} \mathrm{~g} \mathrm{~cm}^{-3}$ ) for the $2 \mathrm{D}$ models G15 (GR, full rates, black thick solid line), S15 (GR, reduced rates, blue, thick, dash-dotted), M15 (pseudo-Newtonian, full rates, red, thick, dashed), and M15 (purely Newtonian, black, thick, dotted). 1D models corresponding to G15, M15, and S15 are also shown as thin lines for comparison. Note that the shock is located considerably further out in S15-1D than in G15-1D and M15-1D. This is a consequence of the strong sensitivity of the shock position $r_{\mathrm{sh}}$ to the PNS radius, $r_{\mathrm{PNS}}$, for a stationary spherical accretion flow $\left(r_{\mathrm{sh}} \propto r_{\mathrm{PNS}}^{8 / 3}\right.$, see, e.g., Equation (1) of Marek \& Janka 2009). The larger PNS radius in S15-1D can in turn be traced to less efficient cooling by $\mu / \tau$ neutrinos and higher temperatures in the density region $10^{12} \ldots 10^{13} \mathrm{~g} \mathrm{~cm}^{-3}$. Different PNS radii (caused by proto-neutron star convection; see Appendix $\mathrm{C}$ in Buras et al. 2006a) are also responsible for the larger shock radii in the 2D models G15 and M15 compared to G15-1D and M15-1D at early times, when multi-dimensional effects in the gain region do not yet play a significant role. (The data for M15-1D have been provided by L. Hüdepohl.) tion $r_{\mathrm{sh}}(\theta)$ into Legendre polynomials up to $l=3$. $a_{\ell}$ is computed as

$$
a_{\ell}=\frac{2 \ell+1}{2} \int_{0}^{\pi} r_{\mathrm{sh}}(\theta) P_{\ell} \mathrm{d} \cos \theta .
$$

Hot-bubble convection then starts $\approx 70 \ldots 90 \mathrm{~ms}$ after bounce and slowly pushes the shock further out than in 1D (see Figure 2). It again provides the seed for fast growth of further SASI activity, but it is only at the time when the $\mathrm{Si} / \mathrm{SiO}$ interface reaches the shock that the SASI really starts to become vigorous (Figure 3). Once these multi-dimensional effects dominate the dynamics, the $11.2 M_{\odot}$ and the $15 M_{\odot}$ progenitor evolve rather differently.

\section{1. $11.2 M_{\odot}$ Model}

\subsubsection{Shock Propagation and Explosion Geometry}

In the case of the $11.2 M_{\odot}$ model, the $\mathrm{Si} / \mathrm{SiO}$-interface reaches the shock $\approx 100 \mathrm{~ms}$ after bounce, and the associated drop in the mass accretion rate leads to strong shock expansion, thus essentially enabling the approach to an the explosion as illustrated by Figure 4. During this phase, the post-shock flow becomes dominated by the low- $\ell$-modes of the SASI (Figure 3). As shown in Figure 6, the buoyant convective plumes that contain neutrino-heated gas merge into 2-3 large bubbles. Around $280 \mathrm{~ms}$ after bounce, the tenuous polar plumes disappear almost completely for a short while. This change of the flow geometry even results in a retraction of the average shock radius for $70 \mathrm{~ms}$ (left panel of Figure [5] red lines). From $350 \mathrm{~ms}$ onward, however, the oscillations of the shock become less violent as it is pushed steadily outward and re-sphericized somewhat by sweeping up mass from the spherical progenitor layers (left panels of Figure 3 and 5). This is also reflected by the normalized Legendre coefficients $a_{\ell} / a_{0}$ of the shock position, which decrease to a level of about $\sim 0.1$ after $450 \mathrm{~ms}$ post-bounce. From around $650 \mathrm{~ms}$ onward, we see positive post-shock velocities along the entire shock front. Towards the end of the simulations, we observe two high-entropy bubbles in the northern and 

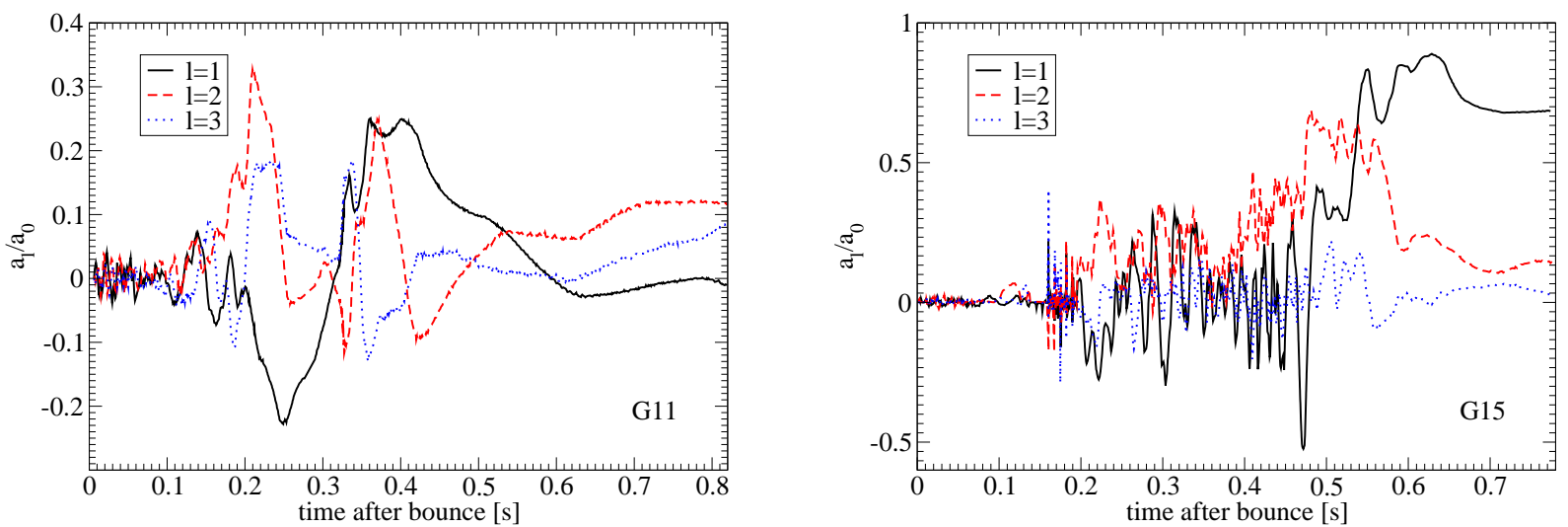

Figure 3. First, second, and third coefficient $a_{1}, a_{2}$, and $a_{3}$, of the decomposition of the shock radius into Legendre polynomials, normalized to the coefficient $a_{0}$ (i.e. the average shock radius) for model G11 (left panel) and model G15 (right panel).
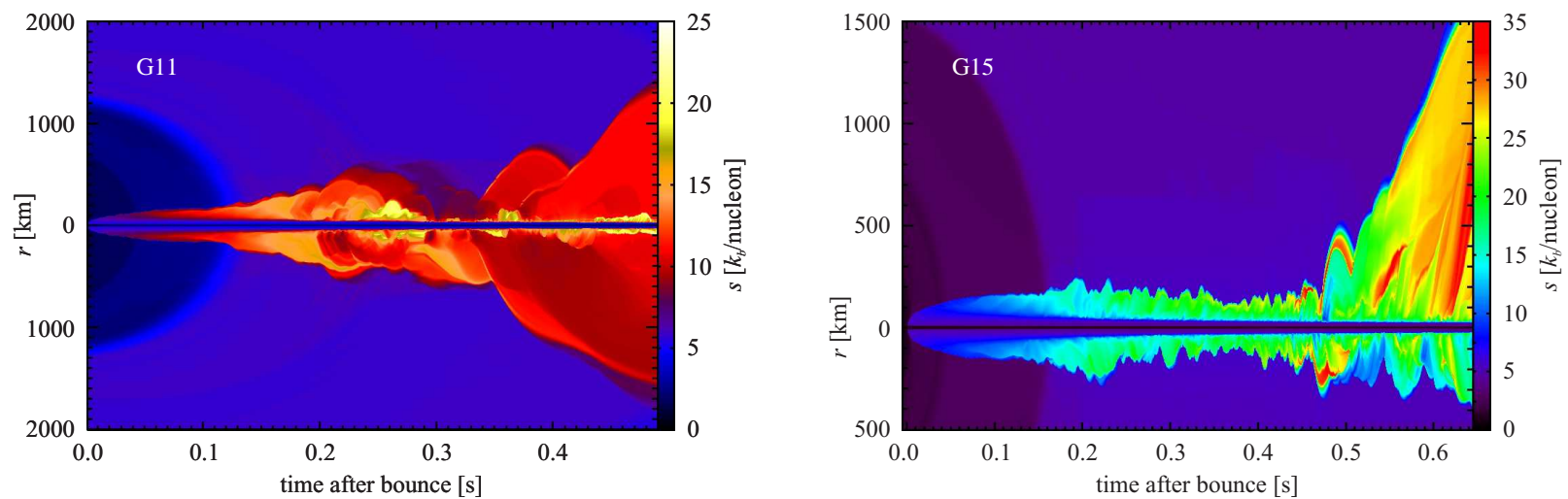

Figure 4. Entropy along the north and south polar axis as a function of time for models G11 (left) and G15 (right). The trajectory of the Si/SiO-interface - initially located at $\approx 1200 \mathrm{~km}$ and $\approx 1800 \mathrm{~km}$, respectively - is visible as a discontinuity in brightness. We point out that model G11 (left) still shows strong SASI activity with repeated phases of shock stagnation or retraction during its long approach to the explosion. It should also be noted that the entropy of the buoyant bubbles pushing the shock further out in the polar directions is quite moderate in this case. Model G15 (right) shows a strong dipolar asymmetry during the explosion phase: While neutrino-heated high-entropy plumes have pushed the shock outward with a propagation velocity of $\gtrsim 10000 \mathrm{~km} \mathrm{~s}^{-1}$ along the northern polar direction, the shock radius in the opposite direction grows much more slowly and non-monotonically. Note that only parts of the simulations are covered by the plots because many features of the earlier phases would not be recognizable on a different scale.
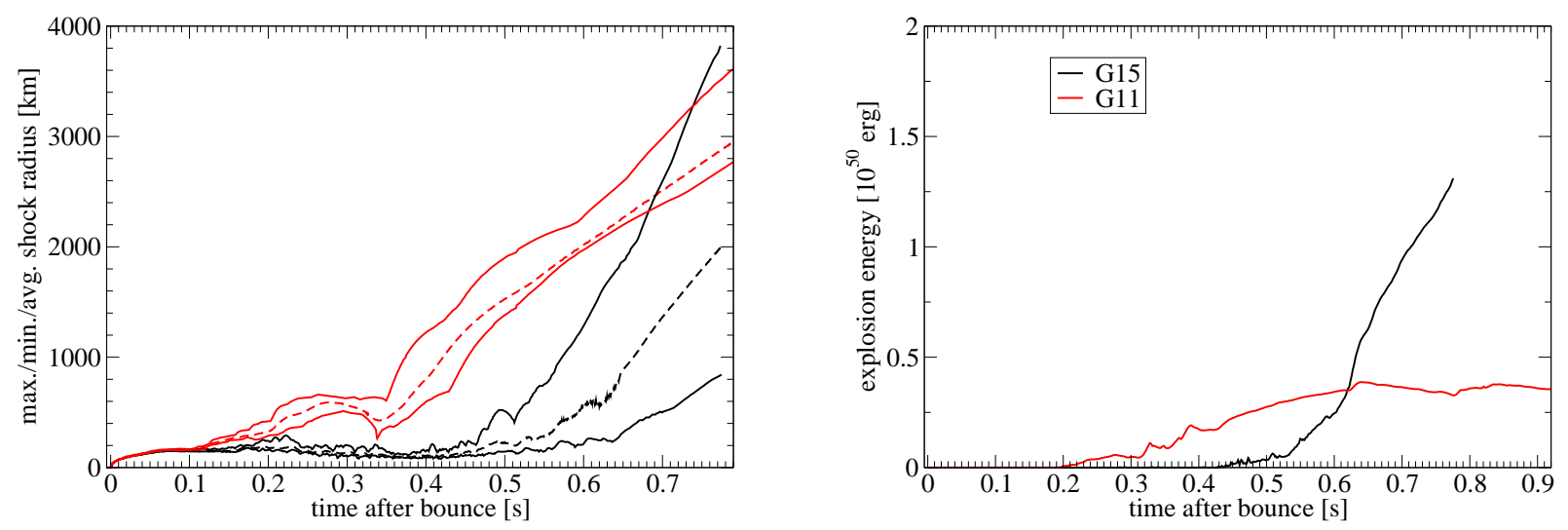

Figure 5. Left panel: Maximum, minimum and average shock radius for models G11 and G15. In both cases, the shock expands and SASI activity increases considerably when the $\mathrm{Si} / \mathrm{SiO}$-interface reaches the shock (at $\approx 100 \mathrm{~ms}$ and $\approx 150 \mathrm{~ms}$, respectively). It recedes again in the case of model G15, and the explosion is launched only several hundreds of ms later. Right panel: The diagnostic "explosion energy" for both models (see text for exact definition). 

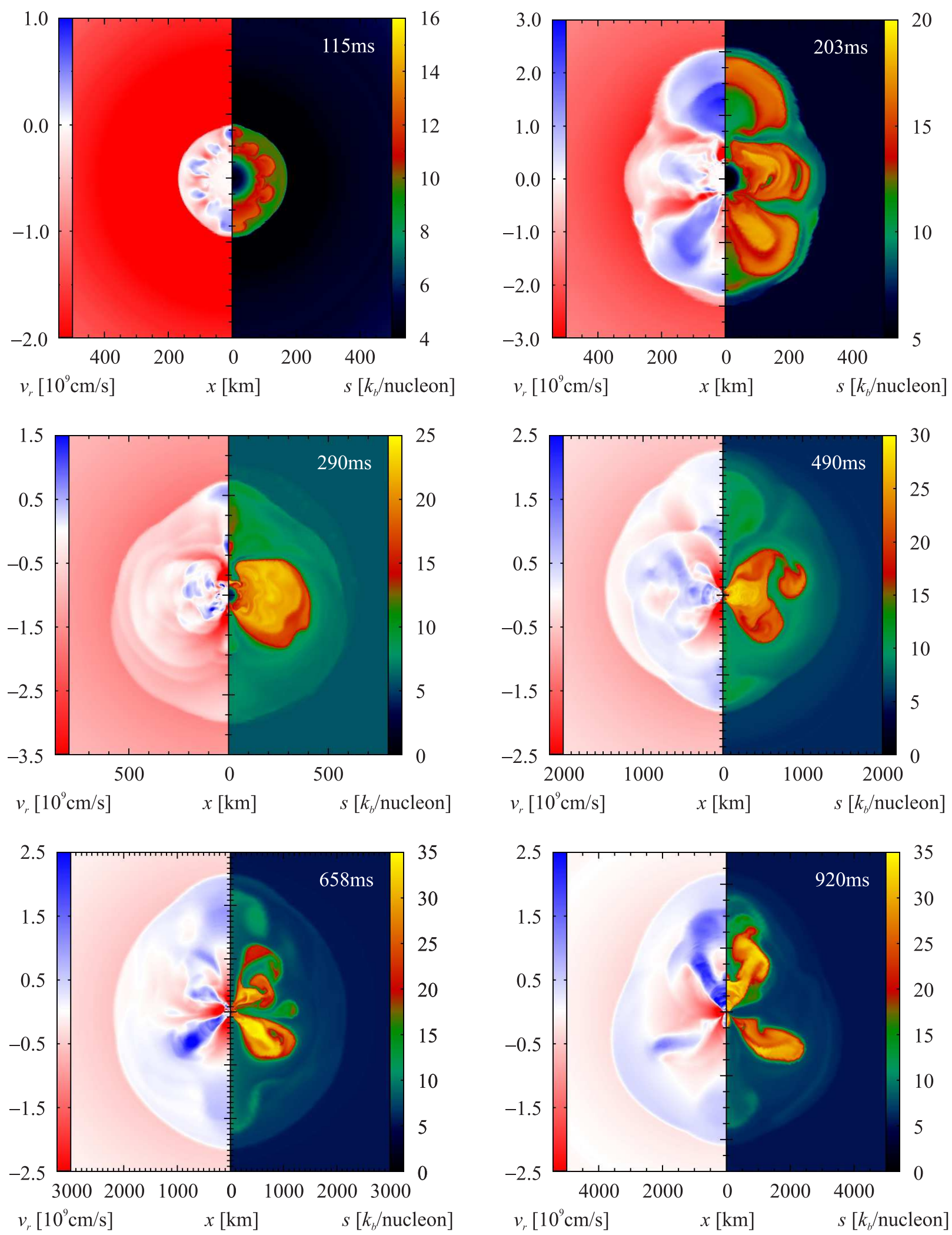

Figure 6. Snapshots of the evolution of model G11, depicting the radial velocity $v_{r}$ (left half of panels) and the entropy per baryon $s$ (right half of panels) $115 \mathrm{~ms}, 203 \mathrm{~ms}, 290 \mathrm{~ms}, 490 \mathrm{~ms}, 658 \mathrm{~ms}$, and $920 \mathrm{~ms}$ after bounce (from top left to bottom right). 


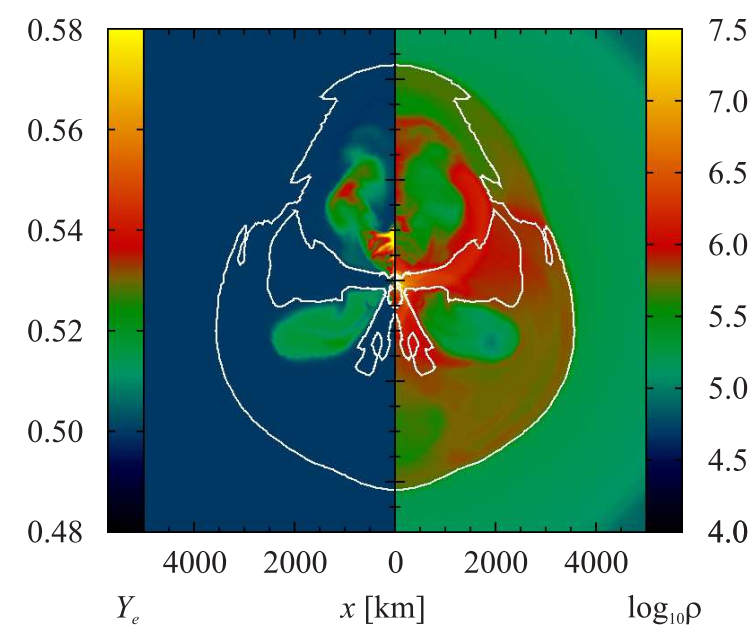

Figure 7. Electron fraction (left half of panel) and density (right half of panel) at a time of $920 \mathrm{~ms}$ after bounce in model G11. The white curves denote the boundary of the region where the local binding energy (Equation 2) is positive, i.e. they enclose the material that is preliminarily classified as ejecta and contains the high- $Y_{e}\left(Y_{e} \approx 0.52\right)$ bubbles of neutrino-heated matter.

southern hemisphere, a rather broad downflow around the equatorial plane, and a further downflow near the south polar axis. At this stage, the deformation of the shock, expressed by the ratio of the maximum and minimum shock radius is still appreciable: $r_{\mathrm{sh}, \max } / r_{\mathrm{sh}, \min } \approx$ $4400 \mathrm{~km} / 3300 \mathrm{~km} \approx 1.3$. Even during the later stages of the simulation, the geometry of the hot plumes does not freeze out, and new downflows may still develop (cp. the snapshots at $490 \mathrm{~ms}$ and $658 \mathrm{~ms}$ ). Accretion onto the proto-neutron star therefore continues until late times, leading to a growth of the baryonic mass of the protonneutron star from $1.275 M_{\odot}$ to $1.36 M_{\odot}$ between $200 \mathrm{~ms}$ and $920 \mathrm{~ms}$.

Figure 3 (left panel) shows that both the dipole $(\ell=$ 1 ) and quadrupole $(\ell=2)$ mode are present in similar strength in model G11, confirming that the deformation of the shock is largest between $200 \mathrm{~ms}$ and $450 \mathrm{~ms}$ after bounce and then decreases to a level of $\sim 0.1$ for the quadrupole during the subsequent evolution.

\subsubsection{Explosion Energy}

We can compute a diagnostic "explosion energy" by integrating over the material with positive binding energy $e_{\text {bind }}$ at a certain time. Since this energy does not account for subsequent nuclear recombination, burning, and the gravitational binding energy of the outer layers of the star, this quantity does not provide a direct measure for the final supernova explosion energy. In the GR case, we define $e_{\text {bind }}$ in terms of the lapse function $\alpha$, the rest-mass density $\rho$, the specific internal energy $\epsilon$, the pressure $P$ and the Lorentz factor $W$ as follows,

$$
e_{\text {bind }}=\alpha\left(\rho\left(c^{2}+\epsilon+P / \rho\right) W^{2}-P\right)-\rho W c^{2} .
$$

In order to maintain consistency with previous studies (Buras et al. 2006a; Marek \& Janka 2009; Bruenn et al. 2009), we exclude rest-mass contributions to the specific internal energy $\epsilon$. It can easily be verified that Equation (2) correctly reduces to

$$
e_{\text {bind }} \rightarrow \rho\left(\epsilon+\rho v^{2} / 2+\Phi\right)
$$

in the Newtonian limit (where $\Phi$ is the gravitational potential) 2 The diagnostic explosion energy is then computed by integrating over the region where $e_{\text {bind }}$ is positive,

$$
E_{\text {expl }}=\int_{e_{\text {bind }}>0} e_{\text {bind }} \mathrm{d} \tilde{V} .
$$

Here, $\mathrm{d} \tilde{V}$ is the three-volume element for the curved space-time metric (and not the flat-space volume element).

The time evolution of $E_{\text {expl }}$ is plotted in the right panel of Figure 5, which shows that material behind the shock first becomes nominally unbound $200 \mathrm{~ms}$ after bounce for model G11. This corresponds to the time when the shock first expands beyond $\sim 400 \mathrm{~km}$, allowing the temperature to drop sufficiently for nucleon recombination to $\alpha$-particles to set in. The diagnostic explosion energy slowly increases rather unsteadily at an average rate of $6 \times 10^{49} \mathrm{erg} \mathrm{s}^{-1}$, and then seems to level off around $3.5 \times 10^{49}$ erg after $600 \mathrm{~ms}$ post-bounce with some residual fluctuations. By the end of simulation, the total mass of the material with positive binding energy amounts to $0.045 M_{\odot}$.

Despite this seeming "saturation" of $E_{\text {expl }}$, no definitive statement about the final explosion energy can be made as yet at this stage, although we can tentatively estimate corrections due to the energy input from nuclear burning in the shock, $E_{\text {burn }}$, the recombination of nucleons into $\alpha$-particles (and possibly further into heavy nuclei), and the deduction of the binding energy $E_{\text {preshock }}$ of the unshocked matter. All of these correction terms are of a similar magnitude as the current diagnostic value $E_{\text {expl }}$ : The binding energy $E_{\text {preshock }}$ of all the material ahead of the shock is roughly $-7.5 \times 10^{49} \mathrm{erg}$ (i.e. exceeding $\left.E_{\text {expl }}\right)$, which would have to be included in the total explosion energy budget if these layers were expelled completely. In reality, part of the pre-shock material will not become unbound but accreted onto the protoneutron star, and the correction to the explosion energy will be smaller, but only a (considerably) longer simulation could provide precise values.

Recombination of nucleons and $\alpha$-particles in the ejecta would provide an additional energy of $E_{\text {rec }} \approx 2 \times 10^{49} \mathrm{erg}$. Burning in the shock will not yield any significant contribution with the current shock velocities as the typical post-shock temperatures are already too low $(<$ $\left.3 \times 10^{9} \mathrm{~K}\right)$ to allow for explosive Si- and O-burning. The uncertainties in these numbers illustrate that in order to obtain a reasonably accurate prediction for the explosion energy of model G11, the simulation probably needs to be extended until the shock reaches the $\mathrm{C}-\mathrm{O}$ shell at $\gtrsim 2 \times 10^{4} \mathrm{~km}$ (at which point the binding energy of the remaining pre-shock matter would be negligible). The final explosion energy depends critically on the fraction of shocked material from this shell that is accreted onto the proto-neutron star and thus need not become unbound at all.

The slow growth and the stagnation of the diagnostic explosion energy visible in Figure 5 at late times is a consequence of relatively inefficient neutrino heating

\footnotetext{
${ }^{2}$ Precisely speaking, we have $\alpha \rightarrow 1+\Phi / c^{2}$ and $W \rightarrow 1+v^{2} / 2$ in the Newtonian limit, and obtain the Newtonian expression as an approximation to $\mathcal{O}\left(\epsilon / c^{2}, P / \rho c^{2}, v^{2} / c^{2}, \Phi / c^{2}\right)$.
} 
in model G11 associated with an unfavorable 2D flow geometry in this case. In the late phases, the rate of energy input by neutrinos in the gain region, $\dot{Q}_{\nu}$, is only $\sim 5 \times 10^{50} \mathrm{erg} \mathrm{s}^{-1}$. With a typical binding energy of a mass element at the gain radius of $\sim 30 \mathrm{MeV} /$ baryon, this implies that only $\sim 0.01 M_{\odot} \mathrm{s}^{-1}$ of additional material can become unbound by neutrino heating. The actual mass flux from the heating region into the ejecta is somewhat higher because recombination of nucleons into $\alpha$-particles also contributes part of the energy for unbinding the newly ejected material. Since the ejecta from the gain region are channeled through relatively narrow outflows into high-entropy bubbles (cp. Figure 6) at high velocities, neutrino heating is rather inefficient due to the short exposure time. This also implies that only a small excess energy - i.e. much smaller than the maximum energy available from recombination of $8.8 \mathrm{MeV} /$ baryon - remains for increasing the total (internal+kinetic+gravitational) energy of the ejecta. Newly ejected material thus adds only a few $10^{49} \mathrm{erg} \mathrm{s}^{-1}$ to the explosion energy. However, the shock also sweeps up material at a rate of about $0.05 M_{\odot} \mathrm{s}^{-1}$, which implies a negative energy flux into the "ejecta region" of $\sim 5 \times 10^{49} \mathrm{erg} \mathrm{s}^{-1}$. This may balance or even exceed the energy carried by fresh ejecta from the gain region, thus accounting for the unsteady evolution of $E_{\text {expl }}$. With energy being fed into the ejecta at such a low rate, a considerable fraction of the material swept up by the shock will be channeled into the downflows and accreted onto the proto-neutron star (Figure 6). We already observe that the accretion rate onto the proto-neutron star starts to increase again towards the end of the simulation, which leads to a late-time rise of the electron neutrino and antineutrino luminosities (which will be discussed in a subsequent paper on the neutrino signal). All these indications suggest that model G11 remains a low-energy case and is likely to represent a fallback supernova, i.e. the shock will propagate through the envelope and initially accelerate the swept-up material to positive velocities, but a large fraction of the shocked material will remain gravitationally bound and eventually fall back onto the neutron star.

\subsubsection{Ejecta Composition}

Although the final ejecta composition for our models can only be determined by detailed nucleosynthesis calculations once the amount of fallback is known, a few remarks can already be made about the nucleosynthesis conditions on the basis of the entropy (Figure 6) and the electron fraction (Figure 7) of the material that is presumably ejected. Low-entropy matter $\left(s \lesssim 10 \mathrm{k}_{b} /\right.$ nucleon) with $Y_{e} \approx 0.5$ that has undergone little or no neutrino heating, but has either been swept up directly by the shock or has been pushed out by neutrino-heated bubbles before falling inward to smaller radii, contributes most of the mass in the "ejecta region". Depending on the maximum temperature reached before expansion sets in, this material has partially been processed by nuclear burning to various degrees: According to the simple "flashing" treatment in the VERTEx code (Rampp \& Janka 2002), intermediate-mass elements dominate the composition.

Hot, neutrino-processed material with entropies of up to $35 \mathrm{k}_{b} /$ nucleon makes up only for a small frac- tion $\left(\sim 0.005 M_{\odot}\right.$ or $\left.\sim 11 \%\right)$ of the material classified as ejecta by the end of the simulation. This part of the ejecta is proton-rich with an electron fraction $Y_{e}$ ranging from $\approx 0.51$ up to $\approx 0.58$. Different from the case of electron-capture supernovae (Wanajo et al. 2011) and unlike Pruet et al. (2005), who considered an artificially triggered $2 \mathrm{D}$ explosion of the $15 M_{\odot}$ progenitor of Woosley \& Weaver (1995), we do not observe any slightly neutron-rich pockets in the ejecta, which is a consequence of the different (slower) outflow dynamics (a detailed analysis will be provided in a forthcoming paper). We therefore expect the nucleosynthesis yields to conform with the established results for proton-rich outflow conditions, i.e. the ejecta composition will be dominated by ${ }^{56} \mathrm{Ni}$ and helium with an admixture of a few rare isotopes $\left({ }^{45} \mathrm{Sc},{ }^{49} \mathrm{Ti}\right.$, and $\left.{ }^{64} \mathrm{Zn}\right)$ with large production factors (Pruet et al. 2005; Fröhlich et al. 2006b). Depending on the neutrino luminosities there may also be potential for $\nu p$-process nucleosynthesis (Fröhlich et al. 2006a; Pruet et al. 2006).

\section{2. $15 M_{\odot}$ Model}

\subsubsection{Shock Propagation and Explosion Morphology}

Different from the $11.2 M_{\odot}$ case, the development of the explosion in model G15 is not immediately connected to the $\mathrm{Si} / \mathrm{SiO}$ interface reaching the shock although the decrease in the accretion rate results in a transient increase of the average shock to $220 \mathrm{~km}$ at $200 \mathrm{~ms}$ after bounce (see Figure 2 and Figure 4) and also in increased SASI activity (Figure 3. right panel). However, $230 \mathrm{~ms}$ after bounce the shock again starts to recede slowly with the average shock radius reaching a minimum value of about $100 \mathrm{~km}$ at $380 \mathrm{~ms}$. During this period, the SASI remains active with strong dipole and quadrupole components (the maximum amplitudes being $a_{1} / a_{0} \approx a_{2} / a_{0} \approx 0.3$, Figure 3, right panel). Around $400 \mathrm{~ms}$, the average shock radius begins to move outward rather steadily (Figure2), and at about $430 \mathrm{~ms}$, some material becomes nominally unbound (Figure 5). Model G15 develops a strongly asymmetric explosion (Figures 4588): By the end of the simulation, the shock has reached $3800 \mathrm{~km}$ in the northern hemisphere, while the minimum shock radius over the only remaining strong downflow in the southern hemisphere is only $850 \mathrm{~km}$ (Figure 5); i.e. the ratio $r_{\max } / r_{\min }$ of the maximum and minimum shock radius is as large as 4.5:1. Snapshots of the developing asymmetric explosion with even more extreme shock deformation during earlier phases of the explosion are shown in Figure 8.

\subsubsection{Explosion Energy}

From 540 ms onward, we observe a much more rapid growth of the diagnostic explosion energy than for model G11. By $770 \mathrm{~ms}$, it has reached a value of $E_{\text {expl }}=$ $1.3 \times 10^{50}$ and still continues to increase at a rate of $\approx 7 \times 10^{50} \mathrm{erg} \mathrm{s}^{-1}$. This rate of increase is roughly consistent with the assumption that neutrino heating at a rate of $(4 \ldots 5) \times 10^{51} \mathrm{erg} \mathrm{s}^{-1}$ (Figure 12) allows an outflow rate from the gain region of $\sim 0.06 M_{\odot} \mathrm{s}^{-1}$ (assuming a binding energy of $\sim 40 \mathrm{MeV}$ /baryon at the gain radius), and that recombination of nucleons into $\alpha$ particles leads to an excess energy of $\sim 8 \mathrm{MeV} /$ baryon that actually contributes to the explosion energy. As for model G11, more material is actually swept up by the 

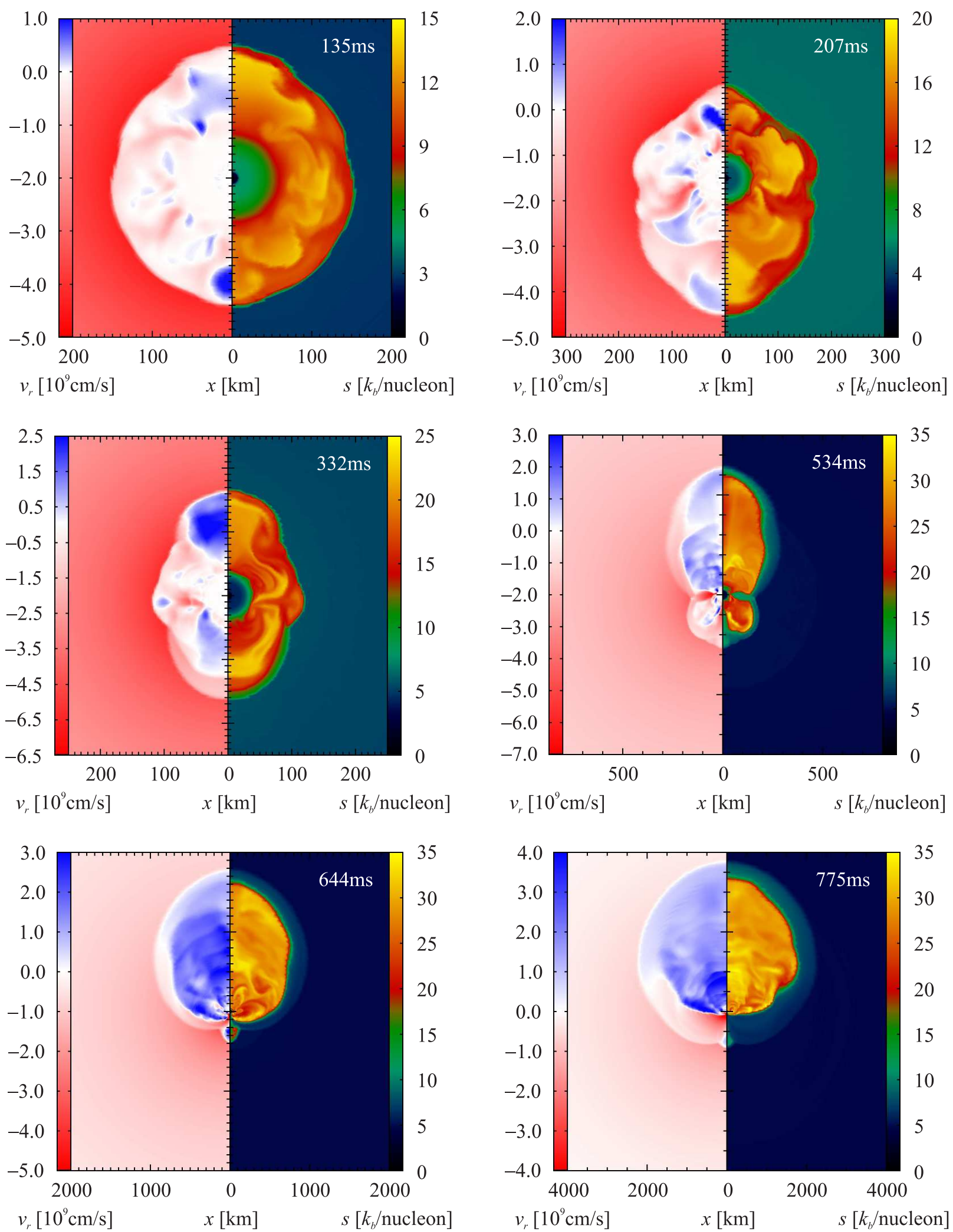

Figure 8. Snapshots of the evolution of model G15, depicting the radial velocity $v_{r}$ (left half of panels) and the entropy per baryon $s$ (right half of panels) $135 \mathrm{~ms}, 207 \mathrm{~ms}, 332 \mathrm{~ms}, 534 \mathrm{~ms}, 644 \mathrm{~ms}$, and $775 \mathrm{~ms}$ after bounce (from top left to bottom right). 


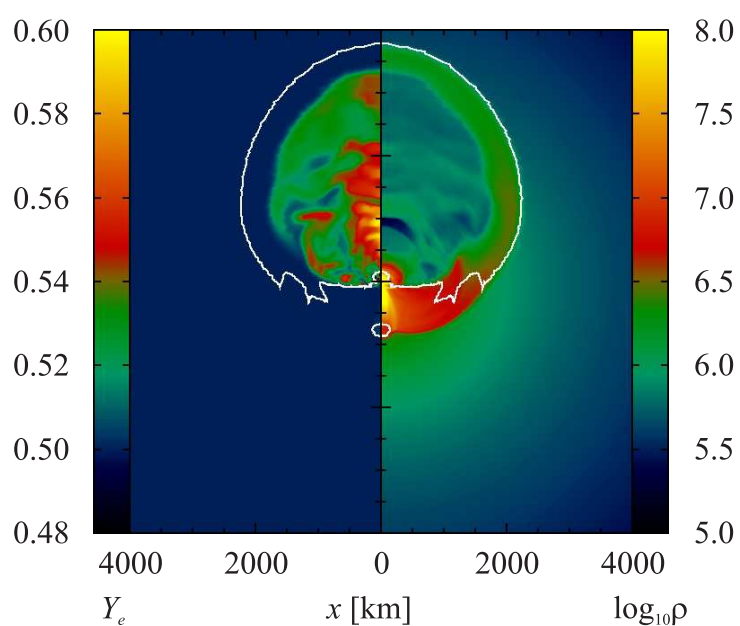

Figure 9. Electron fraction (left half of panel) and density (right half of panel) at a time of $775 \mathrm{~ms}$ after bounce for model G15. The white curves denote the boundary of the region where the local binding energy (Equation 2) is positive, i.e. they enclose the material that is preliminarily unbound and classified as ejecta (mostly located in the northern hemisphere) and contains the high$Y_{e}\left(Y_{e} \approx 0.52\right)$ bubbles of neutrino-heated matter.

shock $\left(\sim 0.15 M_{\odot} \mathrm{s}^{-1}\right)$ than is supplied from the heating region, but in contrast to model G11, the corresponding negative total energy flux into the ejecta region is much smaller $\left(\sim 10^{50} \mathrm{erg} \mathrm{s}^{-1}\right)$ than the positive contribution from hot, neutrino-heated ejecta $\left(\sim 10^{51} \mathrm{erg} \mathrm{s}^{-1}\right) . E_{\operatorname{expl}}$ therefore stably increases for model G15. The presence of a strong and stable downflow in the southern hemisphere is probably helpful for this behavior: Compared to model G11, the accretion rate onto the proto-neutron star is considerably higher $\left(\dot{M} \sim 0.1 M_{\odot} \mathrm{s}^{-1}\right)$ during the late phases, which results in a sizable accretion contribution to the neutrino luminosity and therefore allows for persistently strong neutrino heating in the gain region. Due to continuous accretion, the baryonic mass of the PNS has grown to $1.58 M_{\odot}$ by the end of the simulation.

With respect to the final explosion energy, the same reservations apply as for model G11, i.e. the binding energy of the pre-shock material (roughly $2.6 \times 10^{50} \mathrm{erg}$ ) is still larger than the current value of $E_{\text {expl }}$. However, different from model G11 we expect a significant energy input from burning in the shock on the order of $(1 \ldots 2) \times 10^{50} \mathrm{erg}$ or more, i.e. model G15 has almost reached the stage where the envelope could become unbound completely even without further neutrino heating.

\subsubsection{Ejecta Composition}

The composition of the ejecta in model G15 exhibits some marked differences to model G11, although we can identify the same two components: low-entropy matter with $Y_{e}=0.5$ swept up by the shock, and proton-rich, neutrino-heated matter (Figures 89). Different from model G11, much of the shocked material accumulated by the large expanding bubble in the northern hemisphere has been burnt to ${ }^{56} \mathrm{Ni}$, and even at $775 \mathrm{~ms}$ postbounce, the post-shock temperature is still high enough to allow at least for explosive O-burning. Moreover, hot, neutrino-heated material accounts for a larger fraction of the ejecta $\left(0.01 M_{\odot}\right.$, or roughly one third of the matter with positive total energy). We again find the neutrino-heated ejecta to be exclusively proton-rich, but both the typical and the maximum electron fraction in the neutrino-heated ejecta are even higher than for model G11 with $Y_{e}$ ranging up to 0.6 .

\section{ANALYSIS OF HEATING CONDITIONS AND COMPARISON TO NON-EXPLODING MODELS}

It is interesting to compare the evolution of model G15 to the pseudo-Newtonian run M15, the purely Newtonian model N15, and to simulation S15 with simplified neutrino reaction rates. The average shock radius is relatively similar in all cases until $\approx 400 \mathrm{~ms}$ after bounce (Figure 2), except for model N15, which maintains a larger average shock radius (by $30 \ldots 50 \mathrm{~km}$ ). Among G15, M15, and S15, the shock in model G15 reacts most strongly to the drop in $\dot{M}$ associated with the $\mathrm{Si} / \mathrm{SiO}$ interface and stays a little further out until $380 \mathrm{~ms}$, but the differences between the three models remain rather modest; right before the onset of the explosion in model G15, the shock position is virtually identical. Yet, only a few tens of milliseconds later, an explosion develops in model G15, whereas S15, M15 and even N15 with its relatively large shock radius $(\approx 140 \mathrm{~km})$ show no sign of shock revival. In this section, we discuss the reason for this (unexpectedly) different behavior of the models lacking either the GR hydrodynamics treatment or the full set of neutrino opacities.

\subsection{Analysis Framework}

Shock revival can be understood in terms of a competition of neutrino heating and the downward advection of the gas in the gain layer (e.g. Burrows \& Goshy 1993; Janka 2001). A detailed analysis shows that there is a critical neutrino luminosity for each value of the mass accretion rate $\dot{M}$ of the shock, above which no steady-state accretion solution can exist (Burrows \& Goshv 1993; Janka 2001; Murphy \& Burrows 2008; Pejcha \& Thompson 2012; Fernández 2012). Roughly speaking, conditions become favorable for an explosion if the time the accreted material spends in the gain region (measured by the "advection", "residency", or "dwelling" time-scale $\tau_{\text {adv }}$ ) becomes longer than the time required to lift its binding energy to positive values by neutrino heating (the heating time-scale $\left.\tau_{\text {heat }}\right)$. If a ratio $\tau_{\text {adv }} / \tau_{\text {heat }} \gtrsim 1$ is maintained for a sufficiently long time (typically a few tens of milliseconds), the shock can, in all probability, expand sufficiently to create a positive feedback loop by further increasing $\tau_{\mathrm{adv}}$, thus establishing a runaway situation with sustained shock expansion that eventually leads to an explosion. This concept provides an adequate basis for understanding the different outcome of the exploding models G11 and G15 in contrast to the other $15 M_{\odot}$ runs (cp. Marek \& Janka 2009).

We compute $\tau_{\mathrm{adv}}$ as the ratio of the binding energy $\left|E_{\text {gain }}\right|$ of the material in the gain region and the volumeintegrated energy deposition rate in that region (in agreement with Marek \& Janka 2009 and with the best definition identified by Fernández 2012),

$$
\tau_{\text {heat }}=\frac{\left|E_{\text {gain }}\right|}{\dot{Q}_{\text {heat }}} .
$$

Here, $E_{\text {gain }}$ and $\dot{Q}_{\text {heat }}$ are volume-integrals over the binding energy density $e_{\text {bind }}$ as given by Equation (2) and the local neutrino heating rate per unit volume $\dot{q}_{e}$ between 
the gain radius $r_{\text {gain }}$ (computed from the angle-averaged neutrino heating profile) and the (average) shock radius $r_{\text {shock }}$,

$$
\begin{gathered}
E_{\text {gain }}=\int_{r_{\text {gain }}<r<r_{\text {shock }}} e_{\text {bind }} \mathrm{d} \tilde{V}, \\
\dot{Q}_{\text {heat }}=\int_{r_{\text {gain }}<r<r_{\text {shock }}} \dot{q}_{e} \mathrm{~d} \tilde{V} .
\end{gathered}
$$

There are alternative definitions for the advection timescale $\tau_{\text {adv }}$ (Buras et al. 2006a; Marek \& Janka 2009; Murphy \& Burrows 2008; Peicha \& Thompson 2012), each of which can be supported by very plausible arguments. For the sake of convenience, we choose to express it in terms of two readily available quantities, the accretion rate $\dot{M}$ of gas through the shock and the baryonic rest mass contained in the gain layer $M_{\text {gain }}$,

$$
\tau_{\mathrm{adv}}=M_{\text {gain }} / \dot{M}
$$

where $M_{\text {gain }}$ is given by

$$
M_{\text {gain }}=\int_{r_{\text {gain }}<r<r_{\text {shock }}} \rho W \mathrm{~d} \tilde{V} .
$$

This definition measures the time matter needs to flow through the gain region if steady-state conditions hold, and is used here because it allows for a very straightforward evaluation of $\tau_{\text {adv }} / \tau_{\text {heat }}$ not only in the $1 \mathrm{D}$ case, but also in the multi-dimensional case. The time-scale ratio $\tau_{\text {adv }} / \tau_{\text {heat }}$ was recently also shown by Fernández $(2012)$ to provide a useful instrument for distinguishing models that are going to explode from "pessimistic" ones.

\section{2. $11.2 M_{\odot}$ Progenitor}

The evaluation of the time-scale ratio $\tau_{\text {adv }} / \tau_{\text {heat }}$ confirms that the transition to shock expansion of model G11 is a relatively clear-cut case, and is indeed triggered by the $\mathrm{Si} / \mathrm{SiO}$-interface. As shown by Figure 10, the expansion of the shock due to the drop in the mass accretion rate results in a considerable increase of the advection time-scale $\tau_{\text {adv }}$ (by a factor of $2 \ldots 3$ ) within a few tens of milliseconds, which is sufficient to bring the critical timescale ratio $\tau_{\mathrm{adv}} / \tau_{\text {heat }}$ above unity in conjunction with the support from hot-bubble convection. At later times, $\tau_{\text {adv }} / \tau_{\text {heat }}$ never drops below unity, not even during the transient phase of shock retraction around $300 \mathrm{~ms}$, indicating heating conditions that favor robust shock expansion. These findings are in very good agreement with the pseudo-Newtonian model of Buras et al. (2006a) and Marek \& Janka (2009), even though the explosion morphology is different (spherical instead of dipolar), which is probably the result of stochastic variations of the SASI activity.

\section{3. $15 M_{\odot}$ Progenitor - Influence of General Relativity}

The case of the $15 M_{\odot}$ model is much more interesting because the different outcome of model G15 as opposed to M15, N15, and S15 suggests that this progenitor is a marginal case and may reveal the dependence of the explosion conditions on the input physics used in these three runs, i.e. the influence of the GR treatment and the neutrino reaction rates. Indeed, a comparison of the

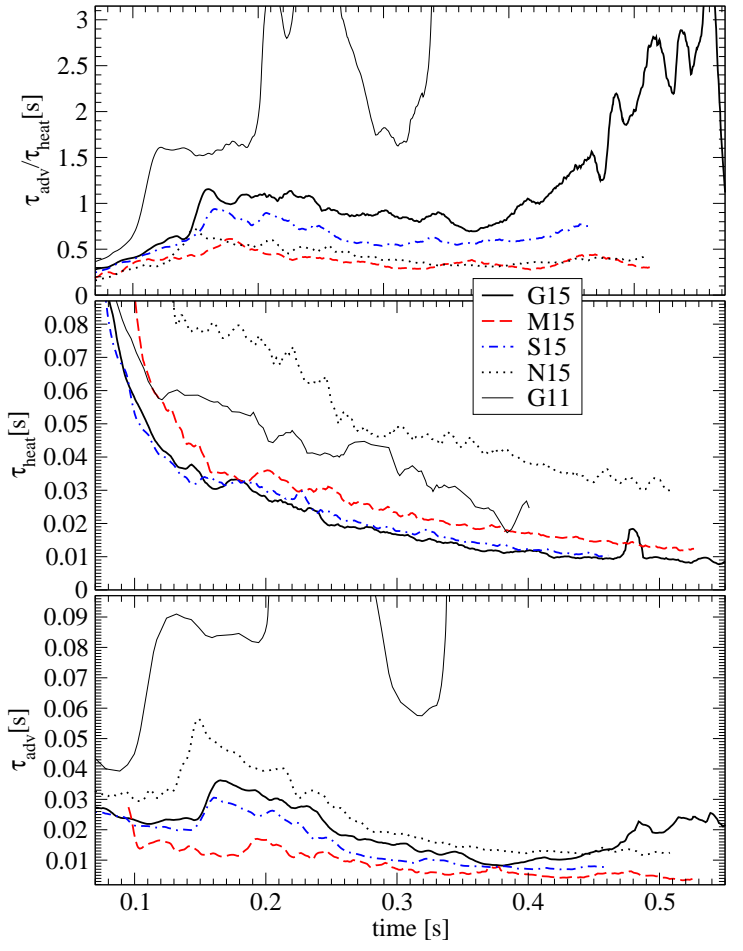

Figure 10. Runaway criterion $\tau_{\text {adv }} / \tau_{\text {heat }}$, heating time-scale $\tau_{\text {heat }}$, and advection time-scale $\tau_{\text {adv }}$ for models G15 (thick black solid line), M15 (thick, red, dashed), S15 (thick, blue, dashdotted), N15 (thick, black, dotted), and G11 (thin solid line, black) smoothed over $10 \mathrm{~ms}$. Note that we do not evaluate the time-scales during the later phase of the explosion when the their definitions are no longer meaningful. Naturally, the time-scales cannot be computed before the formation of the gain layer several tens of milliseconds after bounce either.

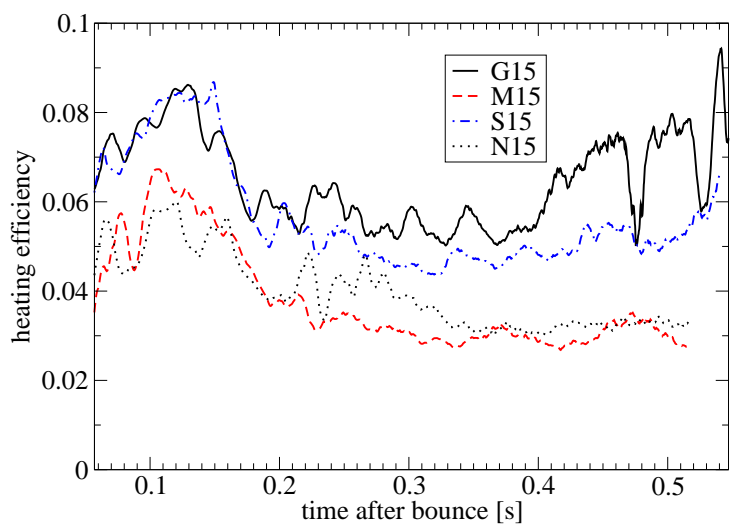

Figure 11. Heating efficiency for models G15 (black solid line), M15 (red, dashed), S15 (blue, dash-dotted), and N15 (black, dotted). The heating efficiency is computed as the ratio $\dot{Q}_{\text {heat }} /\left(L_{\nu_{e}}+\right.$ $\left.L_{\bar{\nu}_{e}}\right)$ of the volume-integrated neutrino heating rate $\dot{Q}_{\text {heat }}$ and the sum of the $\nu_{e}$ and $\bar{\nu}_{e}$ luminosities.

runaway criterion $\tau_{\text {adv }} / \tau_{\text {heat }}$ and the critical time-scales (Figure 10) shows systematic differences: For model G15, we consistently find higher values of $\tau_{\text {adv }} / \tau_{\text {heat }}$ than in the other $15 M_{\odot}$ runs, and this model is also characterized by a larger heating efficiency $\dot{Q}_{\text {heat }} /\left(L_{\nu_{e}}+L_{\bar{\nu}_{e}}\right)$ (Figure 11), i.e. a larger fraction of energy radiated in $\nu_{e}$ and $\bar{\nu}_{e}$ is re-absorbed in the gain layer 3 Moreover,

3 Note that in contrast to Marek \& Janka (2009), we use the luminosities of $\nu_{e}$ and $\bar{\nu}_{e}$ at the gain radius (see Figure 14 instead of the (lower) values for an observer at infinity. 

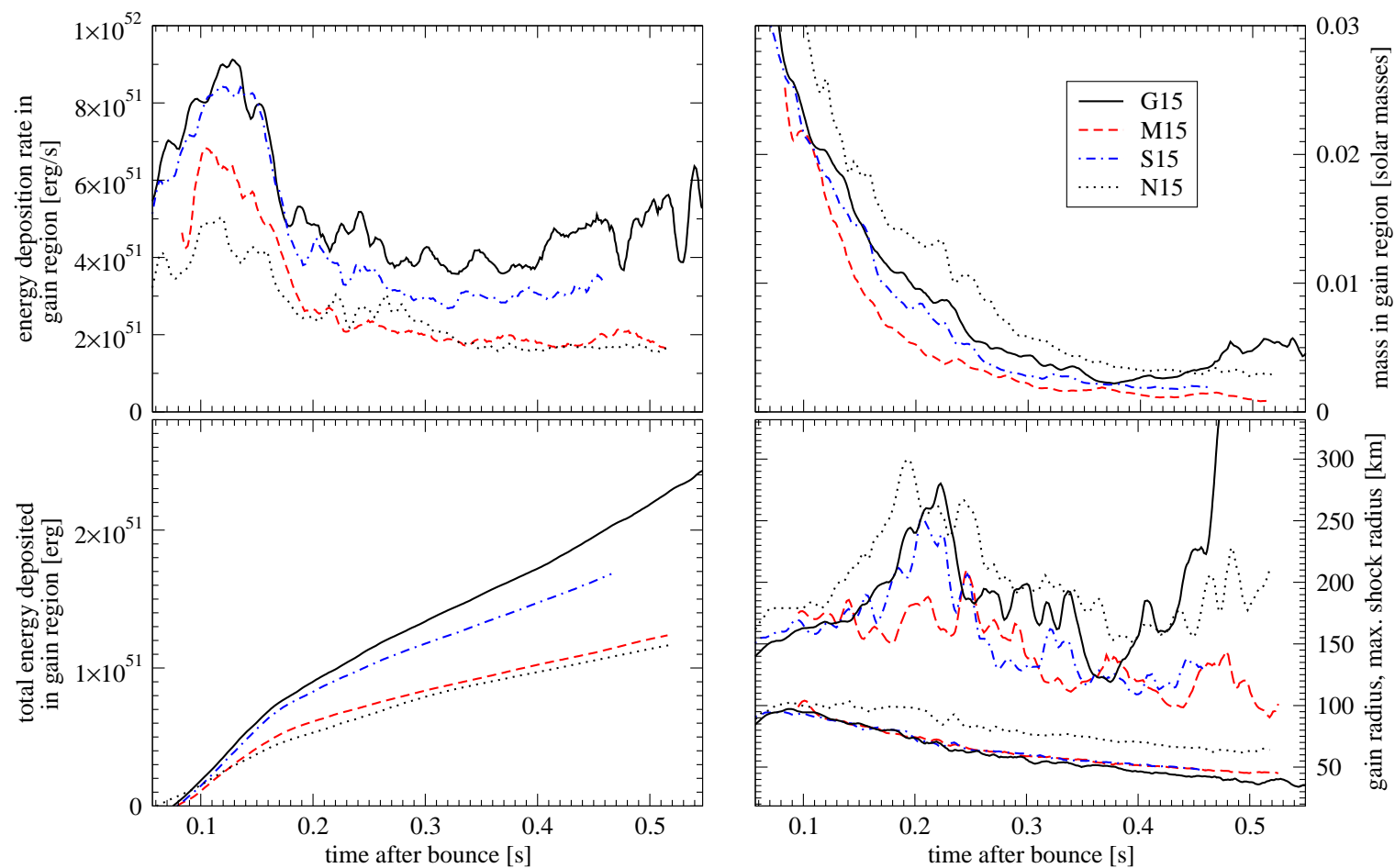

Figure 12. The energy deposition rate in the gain region (top left panel, smoothed over $10 \mathrm{~ms}$ ), the total (time-integrated) energy deposited in the gain region (bottom left), the total mass in the gain region (top right, smoothed over $10 \mathrm{~ms}$ ), the gain radius (bottom right, upper set of lines) and the maximum shock radius (bottom right, lower set of lines)

for models G15 (black solid line), M15 (red, dashed), S15 (blue, dash-dotted), and N15 (black, dotted).

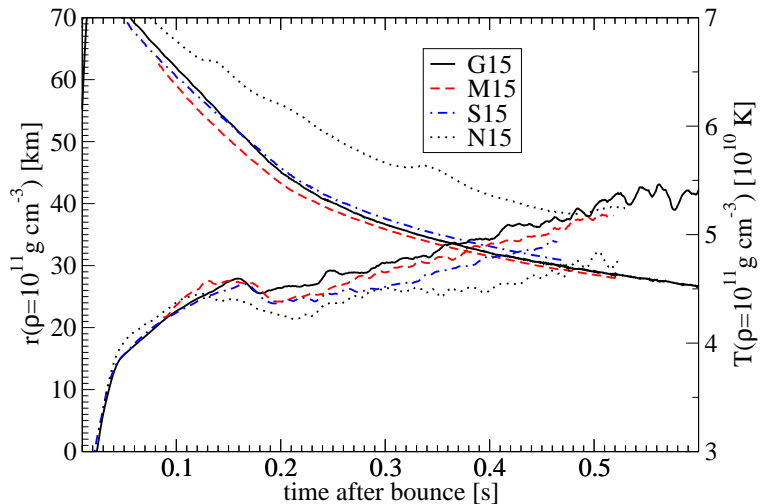

Figure 13. Circumferential radius (decreasing with time) and surface temperature (increasing) of the proto-neutron star for models G15, M15 S15, and N15. The surface is defined by a fiducial density value of $\rho=10^{11} \mathrm{~g} \mathrm{~cm}^{-3}$.

the volume-integrated heating rate $\dot{Q}_{\text {heat }}$ is also highest among the $15 M_{\odot}$ runs (Figure 12).

Compared to model M15, $\tau_{\text {adv }} / \tau_{\text {heat }}$ is typically higher by a factor of two, resulting from the combination of both a longer advection time-scale and a shorter heating time-scale. The purely Newtonian model N15 exhibits a very similar evolution of the runaway criterion as model M15, but this is due to a cancellation of huge differences (amounting to a factor of $2 \ldots 3$ ) in both the advection and the heating time-scale. The relativistic calculation shows, however, that even though the effects of a smaller shock radius (Figure 2) and hence a shorter advection time-scale on the one hand and stronger heating on the other hand partially compensate each other in GR, such a cancellation is not to be taken for granted, and the residual effect, e.g. on the time-scale ratio $\tau_{\text {adv }} / \tau_{\text {heat }}$ may still be on the order of several tens of percents.

For model S15, the time-scale ratio lies about half way in between G15 and N15, suggesting a non-negligible effect of the neutrino microphysics. Considering the claims of Bruenn et al. (2009) to that effect, this is certainly noteworthy, but we shall first turn our attention to the more pronounced differences between the GR model G15 and the runs M15 and N15 with a different treatment of gravity. Since our focus lies on the GR effects in this section, we defer the discussion of model S15 to Section 5.4. however.

\subsubsection{Newtonian Approximation vs. General Relativity}

The purely Newtonian case (N15) stands apart most clearly from the others with large values of $\tau_{\text {adv }}$ and $\tau_{\text {heat }}$, and the reason for this has essentially been given by Bruenn et al. (2001): Due to the shallower potential, the proto-neutron star is considerably more extended in model N15 compared to the GR case (Figure 13), and this also shifts the gain radius and shock radius further out (Figures 2 and [12), thus increasing the mass $M_{\text {gain }}$ in the gain layer. On the other hand, the neutron star surface temperature is also considerably lower compared to GR (Figure 13), resulting in a significant reduction of the neutrino luminosities and mean energies in the gain region (Figure 14) and hence weaker heating in the gain region (Figure 12). Apparently this also leads to less vigorous convection in the purely Newtonian case as the mass-specific kinetic energy contained in non-radial mass motions, $E_{\text {kin }, \theta} / M_{\text {gain }}$, is typically lower than in model G15 (Figure 15) by $40 \%-50 \%$. Stronger convection in GR partly compensates for the reduction of $M_{\text {gain }}$ and $\tau_{\text {adv }}$ due to the smaller neutron star radius and therefore helps to turn the scales in favor of a larger value of the 


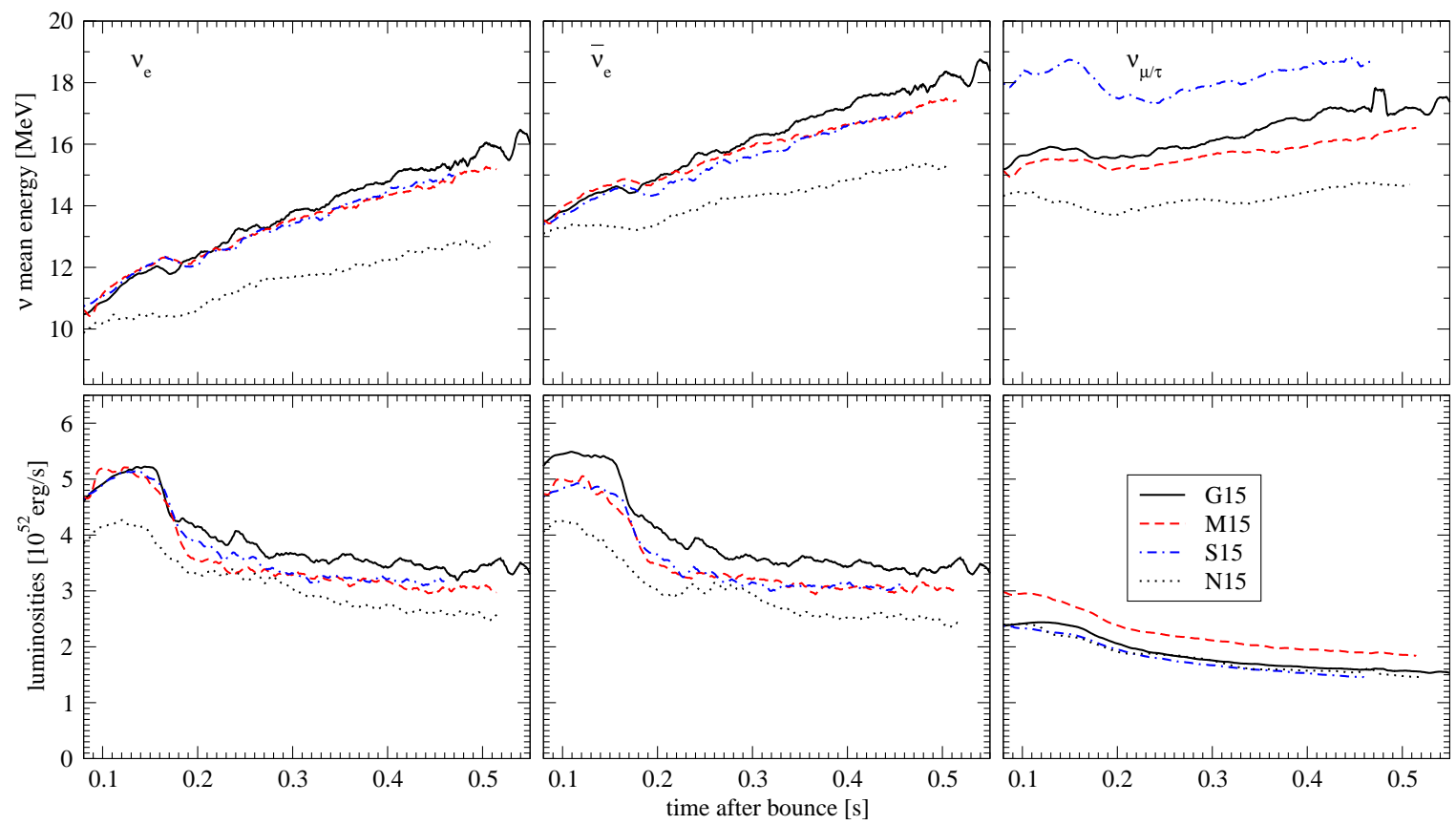

Figure 14. Neutrino mean energies (upper panels) and luminosities (lower panels) at the gain radius for the relativistic model G15 (black solid lines), the pseudo-Newtonian model M15 (red dashed lines), the relativistic model S15 with simplified neutrino rates (blue dash-dotted lines), and the purely Newtonian run N15 (black, dotted). Electron neutrinos, electron antineutrinos, and $\mu / \tau$ neutrinos are shown in the left, middle, and right panel of each row, respectively. The mean energy is defined as the ratio of the angle-averaged neutrino energy and number density, and the luminosity is computed as the integral of the flux over the sphere corresponding to the gain radius. The luminosity given here is for a single species of $\nu_{\mu / \tau}$, not for all four of them.
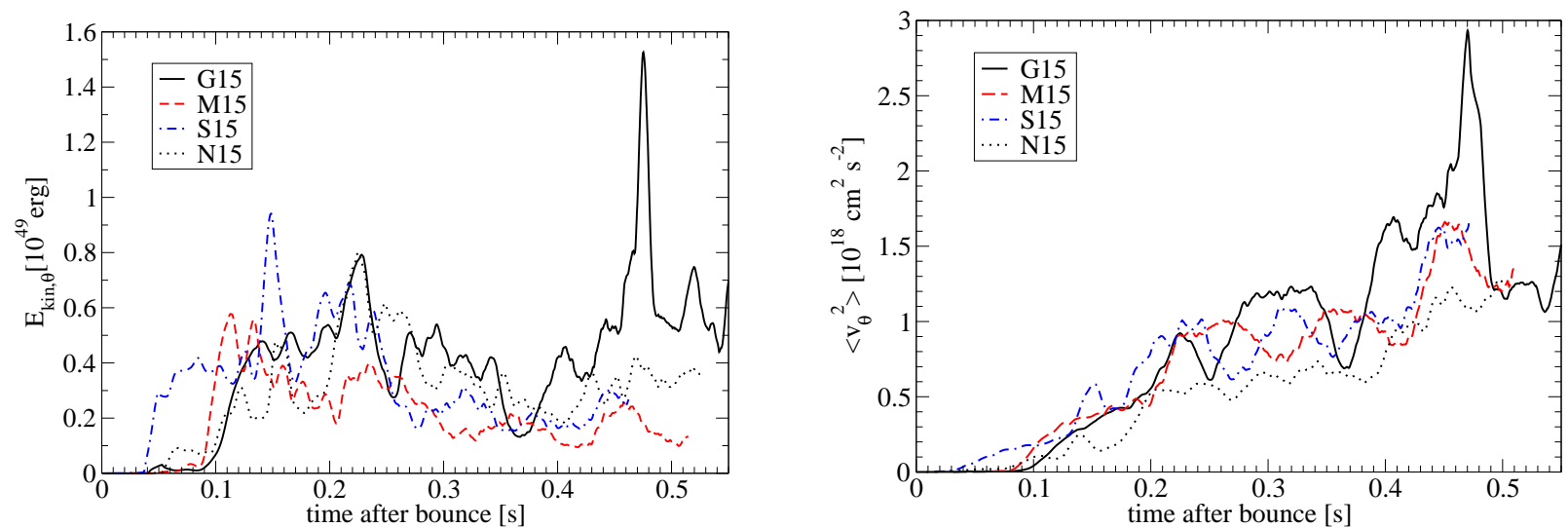

Figure 15. Left: The kinetic energy $E_{\text {kin }, \theta}$ associated with lateral motions in the gain region for models G15 (black solid line), M15 (red, dashed), S15 (blue, dash-dotted), and N15 (black, dotted); the curves are smoothed over $10 \mathrm{~ms} . E_{\text {kin, } \theta}$ can be taken as a possible measure for the combined activity of convection and the SASI, which both contribute to this quantity. Note that a comparison of different models is difficult due to the fact that $E_{\mathrm{kin}, \theta}$ does not only depend on the typical velocities but also on the mass initially available in the gain region. Right: The velocity dispersion $\left\langle v_{\theta}^{2}\right\rangle$ in the gain region, which is related to $E_{\text {kin }, \theta}$ by the relation $\left\langle v_{\theta}^{2}\right\rangle=2 E_{\text {kin }, \theta} / M_{\text {gain }}$. $\left\langle v_{\theta}^{2}\right\rangle$ provides a direct measure for the typical velocities of convective and SASI motions and the violence of these instabilities.

runaway criterion $\tau_{\text {adv }} / \tau_{\text {heat }}$ - the large reduction of $\tau_{\text {heat }}$ emerges as the dominant effect (Figure 10).

In comparing model N15 to G15 and M15, we should also bear in mind that N15 was computed with a higher angular resolution of 128 zones, which might be beneficial for the heating conditions because it was seen to foster explosions in 2D simulations of Hanke et al. (2011). The increase of $\tau_{\text {adv }} / \tau_{\text {heat }}$ in GR compared to the Newtonian approximation may therefore even be underestimated by our analysis.

\subsubsection{Effective Potential Approximation vs. General Relativity}

The comparison between models G15 (GR) and M15 (effective potential) is somewhat more subtle, but the different heating conditions can still be traced back - at least partly - to the neutrino emission from the protoneutron star. Again, the neutrino luminosities and mean energies at the gain radius (Figure 14) turn out to be the crucial factor. In the early phase, the GR run exhibits a noticeable enhancement in the electron antineutrino luminosity (by $\approx 15 \%$ ) and to a lesser extent in the electron neutrino luminosity. In addition, the mean energies of $\nu_{\mathrm{e}}$ and $\bar{\nu}_{\mathrm{e}}$ tend to increase more strongly at late times, with 


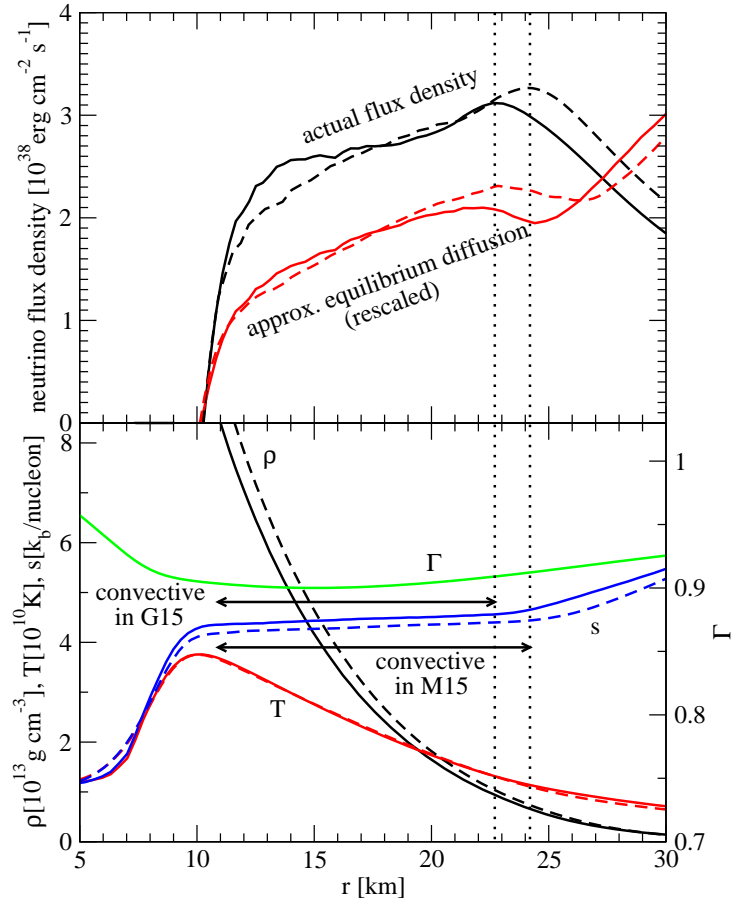

Figure 16. Origin of the different $\nu_{\mu}$ and $\nu_{\tau}$ luminosities in models G15 and M15. The upper panel shows the energy flux density of $\nu_{\mu}$ and $\nu_{\tau}$ at $250 \mathrm{~ms}$ after bounce (black lines) for G15 (solid) and M15 (dashed) and illustrates that a smaller maximum flux is reached at a somewhat smaller "freeze-out" or "number sphere" radius in model G15 (corresponding to a saturation of the luminosity at a lower value). Dotted lines are used to denote the rough location of the freeze-out radius in both models. The lower flux at radii around $24 \mathrm{~km}$ is consistent with an estimate of the flux $H_{\nu \text {, diff }}$ expected for equilibrium diffusion (Equation 10) as indicated by the red curves: While the diffusive fluxes agree well within a large part of the convection zone, the shift of the "dip" in the predicted $H_{\nu \text {,diff }}$ in G15 suggests a lower freeze-out radius and luminosity. Note that the diffusive flux has been rescaled to avoid overlap with the black curve. Since the density- and temperature-dependence of the diffusion coefficient cannot be captured exactly by simple power laws, a perfect fit is not to be expected anyway; Equation (10) is rather used to detect relative differences between the two models G15 and M15. Angle-averaged profiles of the quantities used to evaluate Equation (10), i.e. the density $\rho$, the temperature $T$, and the metric factor $\Gamma$ are shown in the lower panel along with the angleaveraged entropy $s$. The different fluxes in models G15 and M15 stem from several (partially competing) factors, namely a shallower temperature gradient in model G15 from $22 \mathrm{~km}$ outward, which is associated with an earlier rise of $s$ outside the proto-neutron star convection zone, lower densities (which increase the diffusive flux; the relative difference is still $\sim 10 \%$ at $25 \mathrm{~km}$ ), and the reduction of the flux due the $\Gamma$-factor (which is not present in M15). The lower freeze-out radius in model G15 is associated with a different location of the edge of the (roughly adiabatically stratified) PNS convection zone, which is clearly visible in the entropy profile.

the difference reaching almost $1 \mathrm{MeV}$ for the antineutrinos.

Interestingly, the tendency towards slightly more energetic $\nu_{\mathrm{e}}$ 's and $\bar{\nu}_{\mathrm{e}}$ 's in GR is already present in $1 \mathrm{D}$ (see Paper I). This is presumably the result of a slightly different density stratification in GR that cannot be reproduced exactly by the modified Newtonian potential and the approximate GR transport treatment (e.g. due to the identification of coordinate radius and proper radius) used for the M15 run (cp. Marek et al. 2006). The circumferential radius of the PNS (defined as the radius where the density drops to $10^{11} \mathrm{~g} \mathrm{~cm}^{-3}$ ) is indeed larger by $2 \%-4 \%$ in GR, and its surface is somehwat hotter at late times (Figure 13). In contrast to the higher $\nu_{e}$ and $\bar{\nu}_{e}$ luminosi- ties in model G15, the luminosity of $\mu$ and $\tau$ neutrinos is smaller in the GR case. This is the result of general relativistic transport effects and a different stratification at the edge of the PNS convection zone, where the "number sphere" for $\nu_{\mu}$ and $\nu_{\tau}$ (i.e. the sphere up to which production processes and absorption processes remain in equilibrium; see Raffelt 2001; Keil et al. 2003) is located, as we illustrate for a representative snapshot $250 \mathrm{~ms}$ after bounce (Figure 16). The upper panel of Figure 16 shows the energy flux density $H_{\nu}$ of $\nu_{\mu / \tau}$ in the comoving frame for models G15 and M15 at that time, with $H_{\nu}$ rising to somewhat higher values before it decays like $r^{-2}$ outside the "number sphere" 4 To demonstrate that the smaller neutrino flux in the case of model G15 is consistent with the temperature and density stratification depicted in the lower panel of Figure 16 we also show a rough estimate for the diffusive flux for comparison in the upper panel. The diffusive flux $H_{\nu, \text { diff }}$ can be computed as (see, e.g., Pons et al. 1999, Equation 13)

$$
H_{\nu, \text { diff }}=k D \frac{T^{3}}{\alpha}\left(\Gamma \frac{\partial \alpha T}{\partial r}\right)
$$

using Schwarzschild radial coordinates (with $r$ denoting the circumferential radius). Here $\alpha$ is the lapse function, $D$ an appropriate, frequency-averaged diffusion coefficient, and some factors of order unity have been absorbed into the constant $k$ for convenience. A metric factor $\Gamma$ appears because the diffusive flux depends on the derivative $\Gamma \partial / \partial r$ with respect to proper (physical) radius 5 In model M15, no distinction is made between proper radius and circumferential radius $(\Gamma=1)$. As scattering on nucleons is the dominant source of opacity, the diffusion coefficient $D$ is roughly proportional to $\rho^{-1} T^{-2}$ in the density regime that we are considering here. Figure 16 demonstrates that Eq. (10) predicts different fluxes in the region around the "number sphere" and also points to a slightly smaller "number sphere" radius in G15, which is associated with a different location of the outer boundary of the PNS convection zone (Figure 16). The lower flux, mainly due to the factor $\Gamma$ in the GR case and the smaller "number sphere" radius account for the $\sim 20 \%$ lower $\nu_{\mu}$ and $\nu_{\tau}$ luminosity in model G15. On the other hand, there is no such reduction of the neutrino mean energy of $\nu_{\mu}$ and $\nu_{\tau}$ in model G15, because neutrino-electron scattering and non-isoenergetic neutrino-nucleon scattering still allow for energy exchange and thermal equilibration with the medium at larger radii, where the temperature is higher than in model M15.

While GR transport and stratification effects thus seem to account for the different neutrino emission in models G15 and M15 and, because of the higher $\nu_{e}$ and

4 We remark that the luminosity emerging from the "number sphere" is smaller than the black body luminosity by one to two orders of magnitude due to a very small flux factor (see discussion in Janka 1995). Stefan's law is therefore ill-suited for estimating the luminosity of $\nu_{\mu}$ and $\nu_{\tau}$.

${ }^{5}$ In VERTEX-COCONuT, we do not distinguish between heavy flavor neutrinos and antineutrinos, and the chemical potential $\mu_{\nu_{\mu / \tau}}$ therefore vanishes. Consequently, only derivatives of the temperature appear in the diffusion equation.

6 In Vertex-CoCoNuT we work with a different gauge choice for the radial coordinate (isotropic coordinates $r_{\text {iso }}$ ), but the conversion to the familiar Schwarzschild form of the metric is straightforward. $\Gamma$ can be obtained from the conformal factor $\phi$ as $\Gamma=1+2 r_{\text {iso }} \partial \ln \phi / \partial r_{\text {iso }}$. 
$\bar{\nu}_{e}$ luminosities, suggest somewhat better heating conditions, the huge increase of the time-scale criterion in model G15 still needs to be connected to these findings. Merely reducing the heating time-scale by $\approx 20 \%$ in model G15 compared to M15 would not be sufficient to ensure that the runaway condition $\tau_{\text {adv }} / \tau_{\text {heat }}>1$ is met. At $450 \mathrm{~ms}$ the crucial factor distinguishing the relativistic and the pseudo-Newtonian run is the larger value of $M_{\text {gain }}$ (Figure 12) and the longer advection time-scale $\tau_{\text {adv }}=M_{\text {gain }} / \dot{M}$ (Figure 10). Part of this increase in $\tau_{\mathrm{adv}}$ is a direct consequence of the higher neutrino luminosities, as the increase in thermal pressure behind the shock allows for a larger mass in the gain region. Since the position of the gain radius is almost identical in model G15 and M15 (Figure 12), this can be illustrated qualitatively on the basis of a very rough approximation: Requiring balance between heating and cooling at the gain radius with heating and cooling rates per nucleon roughly proportional to $L\left\langle E_{\nu}^{2}\right\rangle / r^{2}$ and $T^{6}$, respectively (e.g. Bethe \& Wilson 1985; Janka 2001), and assuming that the gas at the gain radius is radiation-dominated, we find that the pressure $P_{\text {gain }}$ and the temperature $T_{\text {gain }}$ at the gain radius scale as

$$
P_{\text {gain }}^{3 / 2} \propto T_{\text {gain }}^{6} \propto \frac{L_{\nu}\left\langle E_{\nu}^{2}\right\rangle}{r_{\text {gain }}^{2}} .
$$

The stratification in the gain region is roughly adiabatic with the pressure and density following power laws $\left(P \propto r^{-4}, \rho \propto r^{-3}\right)$, and in spherical symmetry we can therefore approximatively determine the radius of the stagnant accretion shock with the help of the jump conditions at the shock. For the post-shock pressure $P_{\text {post }}$, we have

$$
P_{\text {post }}=\left(1-\frac{1}{\beta}\right) P_{\text {ram }}
$$

where $P_{\text {ram }}$ is the ram pressure ahead of the shock, and $\beta$ is the ratio of the post- and the pre-shock density. $P_{\text {ram }}=\rho_{\text {pre }} v_{\text {pre }}^{2}$ can be computed assuming that the preshock velocity $v_{\text {pre }}$ is a certain fraction of the free-fall velocity, i.e. $v_{\text {pre }} \propto 1 / \sqrt{r_{\mathrm{sh}}}$, and therefore scales as

$$
P_{\text {ram }}=\frac{\dot{M} v_{\text {pre }}}{4 \pi r_{\mathrm{sh}}^{2}} \propto \frac{\dot{M}}{r_{\mathrm{sh}}^{5 / 2}}
$$

On the other hand, the post-shock pressure is related to the pressure at the gain radius roughly by

$$
P_{\text {post }} r_{\text {sh }}^{4} \approx P_{\text {gain }} r_{\text {gain }}^{4} .
$$

Assuming $r_{\text {gain }}$ to be fixed (motivated by Figure 12), the shock radius therefore varies with $L_{\nu}\left\langle E_{\nu}^{2}\right\rangle$ as

$$
r_{\mathrm{sh}} \propto\left(L_{\nu}\left\langle E_{\nu}^{2}\right\rangle\right)^{4 / 9}
$$

Using Equation (15), we can compute the mass $M_{\text {gain }}$ in the gain region for a density stratification with $\rho \propto r^{-3}$,

$$
M_{\text {gain }} \propto \int_{r_{\text {gain }}}^{r_{\mathrm{sh}}} \frac{\beta \dot{M}}{v_{\text {pre }} r_{\text {sh }}^{2}}\left(\frac{r_{\mathrm{sh}}}{r}\right)^{3} r^{2} \mathrm{~d} r \propto \dot{M} r_{\mathrm{sh}}^{3 / 2} \ln \left(\frac{r_{\mathrm{sh}}}{r_{\text {gain }}}\right)
$$

The logarithmic derivative,

$$
\frac{\partial \ln M_{\text {gain }}}{\partial \ln \left(L_{\nu}\left\langle E_{\nu}^{2}\right\rangle\right)} \approx \frac{2}{3}+\frac{4}{9 \ln \left(r_{\text {sh }} / r_{\text {gain }}\right)} \sim 1
$$

can be taken as a measure for the sensitivity of $M_{\text {gain }}$ to changes in $L_{\nu}\left\langle E_{\nu}^{2}\right\rangle$. Although based on a rather crude approximation, Equation (17) suggests that changes in the luminosity and neutrino energy induce comparably large changes in $M_{\text {gain }}$ and hence $\tau_{\mathrm{adv}}$.

In addition, stronger neutrino heating also leads to more violent activity of non-radial mass motions in the form of convection and the SASI (see Figure 15). This in turn increases the residence time-scale of matter in the gain region and thus further boosts the effect of the stronger neutrino heating in model G15. With both mechanisms working in combination, the mass in the gain region $M_{\text {gain }}$ and hence the advection time-scale $\tau_{\text {adv }}$ reach considerably larger values in model G15 (except for a short quiet period (see Figures 42) with little SASI activity between $350 \mathrm{~ms}$ and $400 \mathrm{~ms}$ ). Likewise, the energy deposition rate in the gain region (Figure 12) remains higher than in model M15 by a factor of $\approx 2$ from $200 \mathrm{~ms}$ onward.

While we view all these aspects as a likely explanation, it cannot be excluded that other factors are responsible for the different evolution of the shock after the accretion of the $\mathrm{Si} / \mathrm{SiO}$ and the relatively quiet period around $350 \mathrm{~ms}$. Different growth and saturation properties of the SASI due to general relativistic effects could be invoked as an alternative explanation, although this seems rather far-fetched considering that the non-linear behavior of the SASI is still under investigation even in the Newtonian case (where parasitic Kelvin-Helmholtz or Rayleigh-Taylor instabilities have been proposed as a saturation mechanism by Guilet et al. 2010). The possibility of numerical effects (in a broad sense) ought to be considered more seriously: The hydro solvers CoCoNuT and Prometheus used for model G15 and M15, respectively (Table 1), employ different Riemann solvers, PROMETheUs relies on dimensional splitting, the radial grids are not identical, and neither are the initial perturbations. However, there is little grounds for relating these technical differences to the behavior of models G15 and M15. Apart from the fact that the $\mathrm{Si} / \mathrm{SiO}$ interface is preserved a little more sharply in CoCoNuT (which may account for a slightly stronger reaction of the shock to the reduced accretion rate, Figure 2), we have no tangible evidence for such a connection. Moreover, the fact that the CoCoNuT model S15 - with a less pessimistic ratio $\tau_{\text {adv }} / \tau_{\text {heat }}$ (Figure 10) - also responds less vigorously to the drop in the accretion rate than model G15 points to a physical origin as well. It therefore seems that the more rigorous GR treatment is ultimately the reason for the more optimistic evolution of model G15 compared to the pseudo-Newtonian simulation M15.

\subsubsection{Differences to Previous Pseudo-Newtonian Simulations}

Marek \& Janka (2009) already investigated the $15 M_{\odot}$ progenitor of Woosley \& Weaver (1995) in the framework of the effective potential approximation using the same ray-by-ray variable Eddington factor method that has been applied in our study. Given the close similarity of their model setup, the simulations presented here must also be interpreted against the background of their earlier results.

Marek \& Janka (2009) considered four different simulations of the $15 M_{\odot}$ progenitor, namely two rotating 

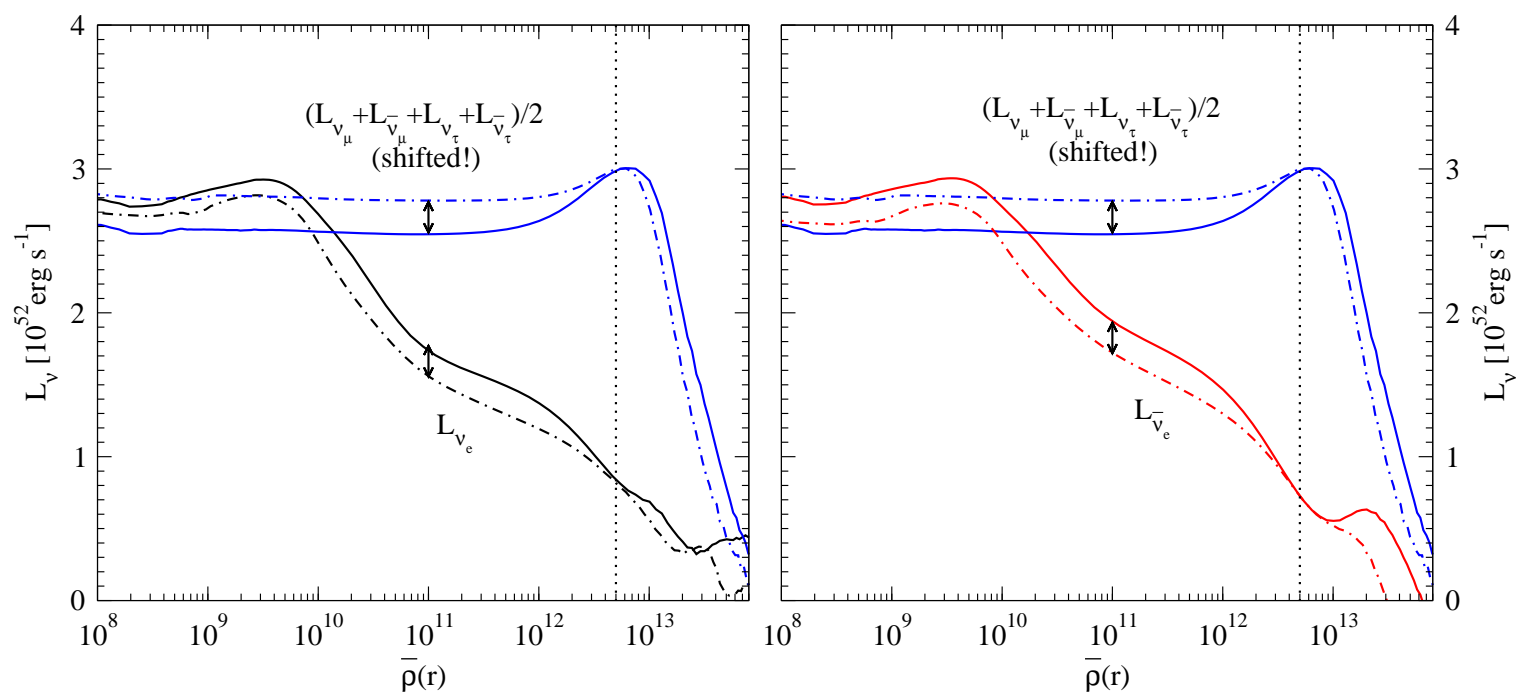

Figure 17. Illustration of enhanced $\nu_{e}$ and $\bar{\nu}_{e}$ emission as an indirect consequence of non-isoenergetic nucleon recoil effects in the $\nu_{\mu} / \tau$ sector. The two panels show the redshifted lab-frame luminosities of $\nu_{e}$ (left panel, black curves) and $\bar{\nu}_{e}$ (right panel, red curves) at a time of $400 \mathrm{~ms}$ after bounce as a function of the average density $\bar{\rho}(r)$ at a given radius $r$ for model G15 (black solid lines, non-isoenergetic nucleon recoil included) and model S15 (dash-dotted lines, without energy losses of neutrinos in nucleon scatteringsq). At $\bar{\rho}(r) \approx 5 \times 10^{12} \mathrm{~g} \mathrm{~cm}^{-3}$ (indicated by the dotted vertical line), the $\nu_{e}$ (and, respectively, the $\bar{\nu}_{e}$ ) luminosities with and without nucleon recoil are roughly identical. For the heavy-flavor neutrinos $\left(\nu_{\mu}, \bar{\nu}_{\mu}, \nu_{\tau}\right.$, and $\left.\bar{\nu}_{\tau}\right)$, we show half the total luminosity of the four species and shift the resulting curves to a value of $3 \times 10^{52} \mathrm{erg} \mathrm{s}^{-1}$ at $\bar{\rho}(r) \approx 5 \times 10^{12} \mathrm{~g} \mathrm{~cm}^{-3}$ (blue curves) in order to illustrate the different amount of energy transfer from $\nu_{\mu} / \tau$ to the medium below this density. Energy transfer from $\nu_{\mu / \tau}$ (reflected by the decrease of $L_{\nu}$ towards lower densities) to the background medium occurs roughly between $5 \times 10^{12} \mathrm{~g} \mathrm{~cm}^{-3}$ and a few $10^{11} \mathrm{~g} \mathrm{~cm}^{-3}$. With non-isoenergetic nucleon recoil, the energy input into the medium is larger, and there is a corresponding enhancement of the $\nu_{e}$ and $\bar{\nu}_{e}$ luminosity in precisely the same region. If the additional energy gained from $\nu_{\mu / \tau}$ scattering were completely transferred into and equally split between $\nu_{e}$ and $\bar{\nu}_{e}$, the expected enhancement of the luminosity would be $2 \times 10^{51} \mathrm{erg} \mathrm{s}^{-1}$ (corresponding to the length of the double arrows). This is in reasonable agreement with the observed values.

models (128 angular zones) with different choices for the effective gravitational potential and different resolution in energy space, and two highly-resolved runs (192 angular zones) without rotation and with two different equations of state. Only one of these, the model including rotation and a gravitational potential that somewhat overestimates strong-field effects (case R of Marek et al. 2006), developed an explosion roughly $550 \mathrm{~ms}$ after bounce.

By combining our findings with those of Marek \& Janka (2009), we arrive at a rather coherent picture. In both cases, the $15 M_{\odot}$ progenitor appears to evolve very close to the explosion threshold, as small model variations were sufficient to bring about an explosion, while other simulations with slightly less favorable showed no signal of shock revival at least until $\gtrsim 400 \mathrm{~ms}$ after bounce. The qualitative similarities between our results and those of Marek \& Janka (2009) go much further: It is remarkable that in both cases the explosion is not linked to the transition of the $\mathrm{Si} / \mathrm{SiO}$ interface through the shock but occurs at a much later stage when the SASI again becomes violent after an intermediate phase of relatively weak activity. In both cases, the most optimistic model is one with stronger neutrino emission than for the "best" effective potential (case A) of Marek et al. (2006), which is either due to the better treatment of GR (for our model G15) or the choice of a stronger effective potential (case $\mathrm{R}$ ) for the explosion model LS-rot of Marek \& Janka (2009)7. In

\footnotetext{
7 The explosion model LS-rot of Marek \& Janka (2009) also includes rotation, which, as these authors found, adversely affects the heating conditions. This mitigates the effect of the overly strong gravitational potential and makes their model explode at a similarly late stage as model G15, although with an extra delay of
}

the light of their earlier results, the beneficial role of GR corrections thus seems all the more plausible.

The work of Marek \& Janka (2009) also provides us with reference runs computed with a higher angular resolution, and thus allow us to address an important limitation of the present first generation of GR neutrino hydrodynamics models. In particular, they discuss a nonrotating effective potential model (LS-2D) corresponding exactly to model M15, but with a higher resolution of 192 angular zones (as compared to 64). For the first $200 \mathrm{~ms}$ after bounce, the shock evolution, as well as the advection and heating time-scales agree remarkably well, while the high-resolution run of Marek \& Janka (2009) shows a somewhat more optimistic evolution afterwards. This suggests that our findings about the beneficial effects of GR will prove robust for two reasons: Differences between the GR model G15 and the effective potential run M15 apparently assert themselves earlier than resolution effects and also appear to be more pronounced. Moreover, the comparison of model M15 and model LS-2D of Marek \& Janka (2009) provides further evidence for the more optimistic evolution of high-angular resolution runs in 2D that has been found by Hanke et al. (2011). It therefore seems likely that high-resolution follow up studies will confirm our present results about explosions in GR.

\subsection{Influence of the Neutrino Interaction Rates}

Besides general relativity, the treatment of the neutrino-matter interactions is another potential factor that can influence the heating conditions and the evolution towards an explosion. Models S15 and G15 provide

$\gtrsim 100 \mathrm{~ms}$. 
a comparison between an older "simplified" set of opacities and an up-to-date treatment. These models serve to illustrate the importance of the neutrino microphysics for the dynamics, thus contributing to an ongoing debate about the necessary level of sophistication in the neutrino treatment (Nordhaus et al. 2010; Lentz et al. 2012b, a). In the following, we present an analysis of the most conspicuous differences between the "simplified" and "full" rates with respect to the dynamics of the accretion phase. Due to the computational cost of multi-dimensional neutrino hydrodynamics simulations, we cannot attempt a systematic study of all individual rates; such a systematic investigation is presently only feasible in $1 \mathrm{D}$, where it has recently been carried out by Lentz et al. (2012a).

Both the shock trajectory (Figure 2) and the timescale ratio $\tau_{\text {adv }} / \tau_{\text {heat }}$ (Figure 10) clearly indicate that model G15 with the full set of rates evolves more optimistically than model S15 computed with the simplified rates. Changing the whole "package" of neutrino interaction rates thus appears to influence the dynamical evolution during the accretion phase quite noticeably. As in Section 5.3. the neutrino emission from the proto-neutron star (Figure 14) provides the clue for understanding these differences.

The most conspicuous effect in model G15 is the reduction of the $\nu_{\mu / \tau}$ mean energies (because of the additional inclusion of non-isoenergetic neutrino-nucleon scatterings, see below). This effect is of little direct relevance for the energy deposition in the gain layer to which $\nu_{\mu}$ and $\nu_{\tau}$ hardly contribute. However, electron neutrinos and antineutrinos are also affected on a smaller scale with some repercussions on the heating in the gain layer. In particular, electron antineutrinos are emitted with higher luminosity (by about 10\%) and - towards the later phases - higher mean energy (by up to $0.8 \mathrm{MeV}$ ) in model G15. For the electron neutrinos, the luminosity enhancement is smaller, and the spectra are only a little harder with the improved set of interaction rates.

However, Figure 10 shows that using the improved opacities results only in a relatively insignificant decrease of the heating time-scale in G15. This small effect is magnified by the increase of the advection time-scale due to a larger average shock radius (see Figure 2 and the discussion in Section 5.3.2), as well as by increased convective and SASI activity (see Figure 15), and therefore still leads to an appreciable difference in the time-scale ratio $\tau_{\text {adv }} / \tau_{\text {heat }}$ between models G15 and S15. The reduced heating in model S15 thus at least results in a considerable delay of a possible explosion in this model.

It remains to be discussed how the improved interaction rates in model $\mathrm{G} 15$ contribute to an enhancement of $\nu_{e}$ and $\bar{\nu}_{e}$ emission. G15 and S15 differ in the treatment of a number of interaction processes (see Table 2). Based on two models, we can naturally pin down only the dominant effect relevant for the change of the heating conditions. The reader should note, however, that all of the individual processes for which we use an improved treatment in model G15 (and not only these) are relevant in their own rights in other contexts, as has already been documented in the literature: The electron capture rates on heavy nuclei of Langanke et al. (2003), e.g., lead to stronger deleptonization during collapse, reduce the mass of the homologous core, and result in the formation of a slightly weaker shock at bounce (Hix et al. 2003). The inclusion of nucleon correlations strongly decreases the opacity at high densities (Burrows \& Sawver 1998, 1999; Pons et al. 1998, 1999) and thus shortens the protoneutron star cooling time considerably Hüdepohl et al. 2009). Neutrino pair conversion can somewhat enhance the emission of $\nu_{\mu}$ and $\nu_{\tau}$ from the proto-neutron star (Buras et al. 2003). We refer the reader to the literature for a more detailed discussion of the influence of the individual interaction processes on the neutrino emission and their impact on the dynamics in 1D. Lentz et al. (2012a), in particular, recently studied the interplay of rate variations in great detail in 1D and gave a succinct summary of possible rate effects.

If the dynamical differences between models G15 and $\mathrm{S} 15$ are to be explained by neutrino rate effects, we need a process that can significantly change - perhaps indirectly - the electron neutrino and antineutrino emission during the accretion phase. As a closer analysis shows, among the improvements of interaction rates listed in Table 2 (which include all the aforementioned processes), only energy transfer from $\mu$ and $\tau$ neutrinos to nucleons by scattering reactions in the PNS surface region (not in the gain region!) can achieve such a change and emerges as the most likely cause of the dynamical differences between S15 and G15. As pointed out by several authors (Janka \& Hillebrandt 1989; Suzuki 1990; Raffelt 2001; Keil et al. 2003), $\nu_{\mu}$ and $\nu_{\tau}$ pass an extended scattering atmosphere (dominated by elastic neutrino-nucleon scattering reactions), which separates the "number sphere" where the production reactions (in our case mainly bremsstrahlung and neutrino-neutrino pair conversion) freeze out, and the "transport sphere" which marks the transition to free streaming. In this intermediate region, mainly nucleon recoil in scattering reactions still allows for a certain amount of energy exchange between the neutrinos and the medium, which reduces the mean energies of $\nu_{\mu}$ and $\nu_{\tau}$ appreciably (Figure 14). The enhancement of the neutrino luminosity due to the inclusion of neutrino pair conversion (Buras et al. 2003) is also largely canceled by this energy exchange (although the number flux of $\nu_{\mu}$ and $\nu_{\tau}$ is still considerably larger in model G15 compared to S15, see also Keil et al. 2003).

Our simulations show that "downscattering" effect on the spectra of $\nu_{\mu / \tau}$ has further consequences if the energy exchange between the neutrinos and the background medium is included self-consistently. Neutrino absorption and emission in the PNS surface region maintain a quasi-steady-state temperature stratification which is determined by the neutrino flux temperature and thus by the temperature of regions deeper in the neutron star (which is similar in models G15 and S15). In order to maintain this stratification, any additional energy input from $\nu_{\mu / \tau}$ at densities around $10^{12} \mathrm{~g} \mathrm{~cm}^{-3}$ must be compensated by a corresponding energy loss in $\nu_{e}$ and $\bar{\nu}_{e}$. As this rather efficient conversion of $\nu_{\mu / \tau}$ "recoil energy" of the nucleons into $\nu_{e}$ and $\bar{\nu}_{e}$ happens in a relatively hot layer, there is also a (minor) enhancement of the mean energies of $\nu_{e}$ and $\bar{\nu}_{e}$. We illustrate this "reshuffling" of energy between the different flavors in Figure 17, which shows that the luminosity decrease in $\nu_{\mu / \tau}$ due the scattering losses to nucleons and the enhancement of the $\nu_{e}$ and $\bar{\nu}_{e}$ luminosity occur in precisely the same density region and correspond well in magnitude. 


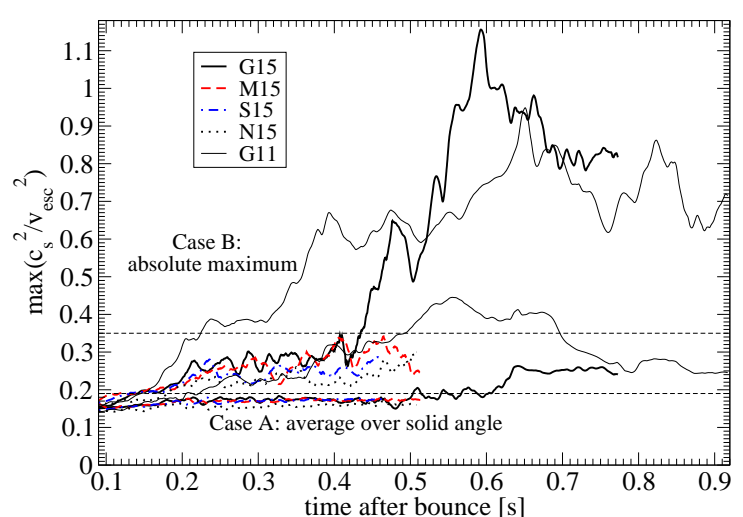

Figure 18. Time evolution of the maximum value of the ratio of the sound speed $c_{s}$ and the (local) escape velocity $v_{\text {esc }}$ for models G11, G15, M15, S15, and N15, smoothed over $10 \mathrm{~ms}$. Two different methods for evaluating $\max \left(c_{s}^{2} / v_{\text {esc }}^{2}\right)$ are used: We either compute spherical averages of $c_{s}^{2}$ before taking the maximum (case A) or take the global maximum (case $\mathrm{B}$ ). Only the region outside the PNS $\left(\rho<10^{11} \mathrm{~g} \mathrm{~cm}^{-3}\right)$ is considered in either case. The escape velocity is computed directly from the gravitational potential or, in the GR case, the lapse function.

It should be emphasized that the additional energy transfer to the medium in the nucleon scattering reactions is crucial for this effect, which cannot be accounted for by the mere change of the scattering and absorption opacity due to the reduction of the final lepton phase space (Schinder 1990; Horowitz 2002). We also point out that the inclusion of the nucleon recoil in the chargedcurrent processes has little effect on the heating by $\nu_{e}$ and $\bar{\nu}_{e}$ : Here, the complete energy of the absorbed neutrino is transferred to the medium anyway - a different partitioning of the deposited energy between nucleons and electrons/positrons does not increase the total energy transfer to the medium. The only change comes from the reduction of the total cross-section (Horowitz 2002), which is small at the relevant neutrino energies between roughly $10 \mathrm{MeV}$ and $20 \mathrm{MeV}$.

All in all, our analysis highlights the need to attend to the details for neutrino-matter interactions in order to model the dynamics of the pre-explosion phase with high accuracy, demonstrating that even some seemingly minor corrections in the rates can have a non-negligible impact on the heating conditions.

\subsection{An Aside on Other Measures for the Criticality of the Accretion Flow}

Extending earlier work on the subject (Burrows \& Goshy 1993; Yamasaki \& Yamada 2007), Peicha \& Thompson (2012) recently re-investigated the problem of a stationary 1D accretion flow onto a proto-neutron star in great detail, and attempted to derive firm criteria for the transition from the phase of quasi-stationary accretion to the explosion. They claimed to have found such a precise and robust criterion in the so-called antesonic condition for the maximum ratio of the local sound speed $c_{s}$ and the escape velocity $v_{\text {esc }}$ in the post-shock region, stating

$$
\max \left(\frac{c_{s}^{2}}{v_{\mathrm{esc}}^{2}}\right)>\frac{3}{16}
$$

as requirement for an explosion. According to their results, this antesonic condition is a more reliable measure for the criticality of the flow than other criteria such as the ratio $\tau_{\text {adv }} / \tau_{\text {heat }}$ used in Section 5, or the requirement of a growing mass and energy in the gain region (Janka 2001). Very recently, Fernández (2012) gave a very careful assessment of the Burrows-Goshy-limit and its relation to the conditions where a steady-state accretion flow in $1 \mathrm{D}$ meets the threshold for a runaway instability.

Considering the ongoing debate about explosion criteria, it is worthwhile to study the applicability of the new antesonic condition to dynamical multi-dimensional simulations of the accretion phase, for which the assumptions of Pejcha \& Thompson (2012) (e.g. spherical symmetry, stationarity) do not necessarily constitute a valid approximation. In order to gauge the usefulness of the new criterion in this context, we need to address two specific questions: First, how sharply does the antesonic condition resolve differences between optimistic and pessimistic models in the accretion phase, and, second, can it accurately capture the onset of the runaway and the development of an explosion? To this end, we plot the time evolution of $\max \left(c_{s}^{2} / v_{\mathrm{esc}}^{2}\right)$ for our 2D models in Figure 18 using two different evaluation methods, viz. averaging $c_{s}$ over solid angle before taking the maximum (Case A), and directly computing the maximum of the ratio $c_{s}^{2} / v_{\text {esc }}^{2}$ in the post-shock region (Case B). As the global maximum is very sensitive to fluctuations and not necessarily indicative for the overall conditions in the gain region, Case A seems more in keeping with the 1D analysis of Pejcha \& Thompson (2012).

Interestingly, we do not observe any clear differences in $\max \left(c_{s}^{2} / v_{\mathrm{esc}}^{2}\right)$ between the $2 \mathrm{D}$ models in Case A prior to the development of an explosion. After an initial growth from 0.1 to $0.17, \max \left(c_{s}^{2} / v_{\text {esc }}^{2}\right)$ settles to a stable average value of about 0.17 in all our simulations of the $15 M_{\odot}$ progenitor. Furthermore, the evolution of $\max \left(c_{s}^{2} / v_{\mathrm{esc}}^{2}\right)$ in $1 \mathrm{D}$ is also virtually indistinguishable from $2 \mathrm{D}$ in the case of the non-exploding model S15 (not shown in Figure 18 as the curves would almost overlap) 8 If we compute the absolute maximum of the velocity ratio of Pejcha \& Thompson (2012) between the proto-neutron star and the shock (case B), we see an increase to values between 0.2 and 0.35 once violent convective and SASI activity starts (as a result of the higher entropy in the convective plumes and SASI lobes). However, a clear hierarchy between the different $15 M_{\odot}$ models is again absent; model M15, which appears to be the most pessimistic case according to the time-scale ratio $\tau_{\text {adv }} / \tau_{\text {heat }}$, even reaches somewhat higher values than model S15 on average.

On the other hand, the onset of the explosion is indeed correlated with an increase in $\max \left(c_{s}^{2} / v_{\text {esc }}^{2}\right)$ beyond 0.2. In Case A this increase can be delayed significantly for a strongly asymmetric explosion (as occurs in run G15). The absolute maximum of $c_{s}^{2} / v_{\text {esc }}^{2}$ appears to be a more robust indicator, but the critical value would have to be quite different from that advocated by

8 High values of $\max \left(c_{s}^{2} / v_{\text {esc }}^{2}\right) \gtrsim 0.15$ are found in all our 1D models. Such high values naturally follow from the jump conditions at the shock for the typical infall velocities in dynamical simulations, which are considerably higher than assumed by Peicha \& Thompson (2012). It is unclear to what extent the choice of the infall velocity or the omission of GR affects the findings of Pejcha \& Thompson (2012). 
Pejcha \& Thompson (2012), namely,

$$
\max \left(c_{s}^{2} / v_{\mathrm{esc}}^{2}\right) \gtrsim 0.35,
$$

but the evidence from two explosion models and two non-exploding models of one of the progenitors is far from conclusive. We find no compelling link between this (incidental?) observation and the arguments of Peicha \& Thompson (2012).

Thus, unlike the time-scale ratio $\tau_{\text {adv }} / \tau_{\text {heat }}$, the antesonic condition does not appear to be a very useful measure for distinguishing optimistic and pessimistic models during the accretion phase, and is of limited use for detecting the runaway situation that ultimately leads to the explosion. There is a number of possible reasons for this apparent conflict with the findings of Peicha \& Thompson (2012). Different from the timescale ratio $\tau_{\text {adv }} / \tau_{\text {heat }}$, the antesonic condition does not make any explicit reference to the heating conditions in the gain layer; it depends only on the pressure, density and gravitational potential at a specific point in the accretion flow (corresponding more or less to the gain radius), which may be crucial for the existence of a stationary accretion flow in spherical symmetry. What happens to such a local quantity beyond the critical point in the dynamical flow of a multi-dimensional situation is unclear, however, whereas $\tau_{\mathrm{adv}} / \tau_{\text {heat }}$ contains global information directly related to the competing influence of accretion and neutrino heating.

Moreover, convection and the SASI alter the structure of the post-shock accretion flow, the heating conditions, and the propagation of the shock considerably in a multidimensional setup. The stratification of the gain layer is not only modified by turbulent mixing; the deviation of the velocity field from that of a stationary spherical accretion flow and the deformation of the shock also become large enough to invalidate many of the assumptions the one-dimensional model of Pejcha \& Thompson (2012) - such as the time-independent, spherically symmetric Euler equations (neglecting, e.g., the turbulent pressure) and the simple form of the jump conditions at the shock (neglecting the SASI). More extreme cases with simultaneous accretion and shock expansion in a dipolar explosion (such as model G15) can hardly be accommodated in a $1 \mathrm{D}$ setup at all. These complications certainly have the potential to change the findings of Peicha \& Thompson (2012) both quantitatively and qualitatively, and to make $\max \left(c_{s}^{2} / v_{\text {esc }}^{2}\right)$ an inferior runaway criterion for multi-dimensional supernova simulations. As demonstrated in the preceding sections, the time-scale ratio $\tau_{\mathrm{adv}} / \tau_{\text {heat }}$ is less affected by such complications and therefore remains, in our opinion, a better diagnostic quantity for the evolution towards the explosion.

\section{SUMMARY AND CONCLUSIONS}

In this paper, we have presented the first multidimensional (2D) general relativistic simulations of supernova explosions with a sophisticated neutrino transport treatment. Using the recently introduced VERTEXCoCoNuT code (Müller et al. 2010), which is based on a relativistic generalization of the variable Eddington factor method for neutrino transport (Rampp \& Janka 2002) combined with the "ray-by-ray-plus" approach for multi-dimensional problems (Buras et al. 2006b;
Bruenn et al. 2006), we calculated the evolution of two progenitors with $11.2 M_{\odot}$ (Woosley et al. 2002) and $15 M_{\odot}$ (Woosley \& Weaver 1995). For the $15 M_{\odot}$ case, we also performed complementary simulations with a pseudo-Newtonian and a purely Newtonian treatment of gravity, and with a simplified set of neutrino interaction rates. We have conducted a comprehensive analysis of the shock propagation, the explosion dynamics, and the heating conditions for these models. With regard to the major goals formulated in Section [1] our results can be summarized as follows:

1. Feasibility of multi-dimensional relativistic supernova simulations: For both the $11.2 M_{\odot}$ and the $15 M_{\odot}$ progenitor, we obtain explosions in $2 \mathrm{D}$ and have been able to extend the simulations several hundreds of milliseconds into the post-bounce and the explosion phase, thus proving the relativistic VERTEX-CoCONuT code to be as robust and stable as its Newtonian counterpart PrometheusVERTEX.

2. Qualitative verification of pseudo-Newtonian results: For the $11.2 M_{\odot}$ progenitor, we confirm the explosions of Buras et al. (2006a) and Marek \& Janka (2009), and for the $15 M_{\odot}$ progenitor, the evolution is very similar to different models computed with the pseudo-Newtonian Prometheus-Vertex code for the first $400 \mathrm{~ms}$ (i.e. before the onset of the explosion in our GR model). As VERTEX-CoCoNuT and Prometheus-VERTEX employ completely independent hydro solvers and somewhat different routines for the moment equations, our results can be viewed as an important step towards code verification in the sense that different codes based on a similar physical model have been shown to produce similar results. However, the different physics leads to important quantitative differences (see next item).

3. Role of general relativistic effects in the supernova problem: For the $15 M_{\odot}$ progenitor of Woosley \& Weaver (1995), we performed a comparison of a relativistic, a purely Newtonian, and a pseudo-Newtonian simulation in order to determine the importance of GR effects during the post-bounce phase. We find significant differences on the order of several tens of percent in some explosion-relevant quantities with a tendency towards more optimistic heating conditions in the GR case. This diagnosis is confirmed by the fact that we observe an incipient explosion in the GR run at $\sim 400 \mathrm{~ms}$, whereas the Newtonian and pseudoNewtonian models fail to explode, at least until $\sim 500 \mathrm{~ms}$ after bounce. Different thermodynamic conditions in the neutrinospheric region have been identified as the most likely cause for the more optimistic evolution in GR. It is noteworthy that the pseudo-Newtonian approach - despite a much better quantitative agreement with the GR model than for the Newtonian run - still leads to a different outcome than the GR case. Interestingly, our GR result yields a similar (successful) late explosion as obtained by Marek \& Janka (2009) or a 
pseudo-Newtonian $15 M_{\odot}$ model including rotation and a gravitational potential that has been found to somewhat overestimate GR corrections in spherical symmetry.

Thus, contrary to claims in the literature (Nordhaus et al. 2010), general relativity may class as a "tens of percent" effect with a similar impact on the dynamics as dimensionality (Nordhaus et al. 2010; Hanke et al. 2011; Takiwaki et al. 2012) and the equation of state (Marek et al., in preparation). GR thus emerges as a key ingredient for accurate supernova simulations.

4. Influence of the neutrino physics input: Based on a $15 M_{\odot}$ model computed with a simplified set of opacities, we also illustrated the sensitivity of the neutrino heating conditions to the neutrino interaction rates. Specifically, we found that the energy transfer from $\nu_{\mu / \tau}$ to the background medium in the neutrinospheric region by nucleon recoil has a beneficial effect, since it effectively provides a means of "converting" a few $10^{51} \mathrm{erg} \mathrm{s}^{-1}$ from $\nu_{\mu / \tau}$ into $\nu_{e}$ and $\bar{\nu}_{e}$, which can then actively contribute to the heating in the gain layer. Without the improved opacities, we observe a considerable delay of the explosion in the $15 M_{\odot}$ case for at least another $>50-100 \mathrm{~ms}$ compared to the onset of the explosion in the baseline model G15 at $\sim 400 \mathrm{~ms}$. Our findings further substantiate claims (Bruenn et al. 2009) about the importance of state-of-the-art neutrino physics input for supernova simulations, although the effect appears to be smaller than that of GR.

On the whole, the inclusion of general relativity in supernova models has turned out to be much more than a marginal improvement, even compared to the effective potential approach which has hitherto been used by some groups (Rampp \& Janka 2002; Marek et al. 2006; Bruenn et al. 2009; Scheidegger et al. 2008). The purely Newtonian approach is definitely ruled out as a viable basis for quantitatively accurate models of core-collapse supernovae. GR may prove more important than expected for accurately capturing the physics of neutrino-driven explosions if the $15 M_{\odot}$ progenitor is any indication. In a subsequent publication, we will demonstrate that GR also has a large impact on the observational signatures such as neutrinos and gravitational waves from supernova cores.

This adds another interesting facet to the supernova problem and probably indicates that the explosion mechanism does not hinge on a single dominating factor after all. General relativity, neutrino microphysics, the equation of state (Marek \& Janka 2009), and dimensionality effects (Nordhaus et al. 2010; Hanke et al. 2011; Takiwaki et al. 2012) may equally contribute to fill the missing parts of the current picture.

Such a situation naturally opens several avenues for future research efforts. Above all, the potentially beneficial effects of GR need to be confirmed by highresolution studies (although improved angular resolution will, in all probability, only lead to more robust explosions in 2D, cp. Hanke et al. 2011). In the long run, a comprehensive and self-consistent approach to supernova modeling, covering the essential aspects of the problem (such as neutrino transport, general relativity, 3D hydrodynamics) with the help of highly accurate numerical algorithms, will be indispensable for a firm quantitative understanding of core-collapse supernova explosions. VERTEX-COCONuT provides a possible platform for further developments in that direction, e.g. the extension of the code to $3 \mathrm{D}$, or the inclusion of more accurate multiangle transport. On the other hand, a more thorough understanding of the individual components of the supernova problem (interplay of SASI and convection, critical explosion conditions, nucleosynthesis, neutron star kicks and spins, etc.) is equally important, and this is no less true for the effects of general relativity discussed in this paper. Having confined ourselves to two progenitors with $11.2 M_{\odot}$ and $15 M_{\odot}$, which produce neutron stars of moderate compactness, we believe that one of the essential tasks will be that of probing deeper into the strong-field regime, where general relativity can be expected to play an even greater role. In our view, the case of more massive progenitors, possibly on the verge to black hole formation, therefore merits particular attention in the future.

We thank A. Bauswein for providing us with Figure 1 and L. Hüdepohl for data from an up-to-date 1D run obtained with the effective potential approximation. This work was supported by the Deutsche Forschungsgemeinschaft through the Transregional Collaborative Research Centers SFB/TR 27 "Neutrinos and Beyond" and SFB/TR 7 "Gravitational Wave Astronomy" and the Cluster of Excellence EXC 153 "Origin and Structure of the Universe" (http://www.universe-cluster.de). The computations were performed on the IBM p690 and the SGI Altix 3700 of the Computer Center Garching (RZG), the NEC SX-8 at the HLRS in Stuttgart, and on the JUMP and JUROPA systems at the John von Neumann Institute for Computing (NIC) in Jülich.

\section{REFERENCES}

Baron, E., Myra, E. S., Cooperstein, J., \& van den Horn, L. J. 1989, ApJ, 339, 978

Bethe, H. A., \& Wilson, J. R. 1985, ApJ, 295, 14

Blondin, J. M., \& Mezzacappa, A. 2006, ApJ, 642, 401

—. 2007, Nature, 445, 58

Blondin, J. M., Mezzacappa, A., \& DeMarino, C. 2003, ApJ, 584, 971

Bruenn, S. W. 1985, ApJS, 58, 771

Bruenn, S. W., De Nisco, K. R., \& Mezzacappa, A. 2001, ApJ, 560,326

Bruenn, S. W., Dirk, C. J., Mezzacappa, A., Hayes, J. C. Blondin, J. M., Hix, W. R., \& Messer, O. E. B. 2006 , J. Phys. Conf. Ser., 46, 393

Bruenn, S. W., \& Mezzacappa, A. 1997, Phys. Rev. D, 56, 7529

Bruenn, S. W., Mezzacappa, A., Hix, W. R., Blondin, J. M., Marronetti, P., Messer, O. E. B., Dirk, C. J., \& Yoshida, S. 2009, Journal of Physics Conference Series, 180, 012018

Buras, R., Janka, H.-T., Keil, M. T., Raffelt, G. G., \& Rampp, M. 2003, ApJ, 587, 320

Buras, R., Janka, H.-T., Rampp, M., \& Kifonidis, K. 2006a, A\&A, 457, 281

Buras, R., Rampp, M., Janka, H.-T., \& Kifonidis, K. 2006b, A\&A, 447, 1049

Burrows, A., Dessart, L., Livne, E., Ott, C. D., \& Murphy, J. 2007a, ApJ, 664, 416

Burrows, A., \& Goshy, J. 1993, ApJ, 416, L75+

Burrows, A., Hayes, J., \& Fryxell, B. A. 1995, ApJ, 450, 830 
Burrows, A., Livne, E., Dessart, L., Ott, C. D., \& Murphy, J. 2006, ApJ, 640, 878

-. 2007b, ApJ, 655, 416

Burrows, A., \& Sawyer, R. F. 1998, Phys. Rev. C, 58, 554

-. 1999, Phys. Rev. C, 59, 510

Carter, G. W., \& Prakash, M. 2002, Physics Letters B, 525, 249

Colgate, S. A., \& White, R. H. 1966, ApJ, 143, 626

Cordero-Carrión, I., Cerdá-Durán, P., Dimmelmeier, H., Jaramillo, J. L., Novak, J., \& Gourgoulhon, E. 2009,

Phys. Rev. D, 79, 024017

Dasgupta, B., O'Connor, E. P., \& Ott, C. D. 2012, Phys. Rev. D, 85, 065008

Demorest, P. B., Pennucci, T., Ransom, S. M., Roberts, M. S. E., \& Hessels, J. W. T. 2010, Nature, 467, 1081

Dimmelmeier, H., Font, J. A., \& Müller, E. 2002, A\&A, 388, 917

Dimmelmeier, H., Novak, J., Font, J. A., Ibáñez, J. M., \& Müller, E. 2005, Phys. Rev. D, 71, 064023:1

Dimmelmeier, H., Ott, C. D., Janka, H.-T., Marek, A., \& Müller, E. 2007a, Phys. Rev. Lett., 98, 251101:1

Dimmelmeier, H., Ott, C. D., Janka, H.-T., Marek, A., \& Müller, E. 2007b, ArXiv e-prints, astro-ph/0705.2675

Dimmelmeier, H., Ott, C. D., Marek, A., \& Janka, H.-T. 2008, Phys. Rev. D, 78, 064056:1

Einfeldt, B. 1988, SIAM J. Numer. Anal., 25, 294

Fernández, R. 2010, ApJ, 725, 1563

—. 2012, ApJ, 749, 142

Fernández, R., \& Thompson, C. 2009, ApJ, 703, 1464

Foglizzo, T., Galletti, P., Scheck, L., \& Janka, H.-T. 2007, ApJ, 654, 1006

Foglizzo, T., Scheck, L., \& Janka, H.-T. 2006, ApJ, 652, 1436

Fröhlich, C., Martínez-Pinedo, G., Liebendörfer, M., Thielemann, F., Bravo, E., Hix, W. R., Langanke, K., \& Zinner, N. T. 2006a, Physical Review Letters, 96, 142502

Fröhlich, C., et al. 2006b, ApJ, 637, 415

Fuller, G. M., Fowler, W. A., \& Newman, M. J. 1982, ApJS, 48, 279

Guilet, J., Sato, J., \& Foglizzo, T. 2010, ApJ, 713, 1350

Hammer, N. J., Janka, H., \& Müller, E. 2010, ApJ, 714, 1371

Hanke, F., Marek, A., Müller, B., \& Janka, H.-Th. 2011, ArXiv e-prints, 1108.4355

Hannestad, S., \& Raffelt, G. 1998, ApJ, 507, 339

Hebeler, K., Lattimer, J. M., Pethick, C. J., \& Schwenk, A. 2010, Physical Review Letters, 105, 161102

Hempel, M., Fischer, T., Schaffner-Bielich, J., \& Liebendörfer, M. 2012, ApJ, 748, 70

Herant, M., Benz, W., \& Colgate, S. 1992, ApJ, 395, 642

Herant, M., Benz, W., Hix, W. R., Fryer, C. L., \& Colgate, S. A. 1994, ApJ, 435, 339

Hix, W. R., Messer, O. E., Mezzacappa, A., Liebendörfer, M., Sampaio, J., Langanke, K., Dean, D. J., \& Martínez-Pinedo, G. 2003, Physical Review Letters, 91, 201102

Horowitz, C. J. 1997, Phys. Rev. D, 55, 4577

-. 2002, Phys. Rev. D, 65, 043001

Hüdepohl, L., Müller, B., Janka, H., Marek, A., \& Raffelt, G. G. 2009, Phys. Rev. Lett.

Isenberg, J. A. 1978, University of Maryland Preprint, ArXiv e-print gr-qc/0702113

Itoh, N., Asahara, R., Tomizawa, N., Wanajo, S., \& Nozawa, S. 2004, ApJ, 611, 1041

Iwakami, W., Kotake, K., Ohnishi, N., Yamada, S., \& Sawada, K. 2008, ApJ, 678, 1207

—. 2009, ApJ, 700, 232

Janka, H.-T. 1995, Astroparticle Physics, 3, 377

-. 2001, A\&A, 368, 527

Janka, H.-T., \& Hillebrandt, W. 1989, A\&AS, 78, 375

Janka, H.-T., \& Keil, W. 1998, in Supernovae and cosmology, ed. L. Labhardt, B. Binggeli, \& R. Buser, 7

Janka, H.-T., Kifonidis, K., \& Rampp, M. 2001, in Lecture Notes in Physics, Berlin Springer Verlag, Vol. 578, Physics of Neutron Star Interiors, ed. D. Blaschke, N. K. Glendenning, \& A. Sedrakian, 363

Janka, H.-T., \& Müller, E. 1996, A\&A, 306, 167

Keil, M. T., Raffelt, G. G., \& Janka, H.-T. 2003, ApJ, 590, 971

Langanke, K., Martínez-Pinedo, G., Müller, B., Janka, H.-T., Marek, A., Hix, W. R., Juodagalvis, A., \& Sampaio, J. M. 2008, Phys. Rev. Lett., 100, 011101:1

Langanke, K., et al. 2003, Physical Review Letters, 90, 241102
Lattimer, J. M., \& Swesty, F. D. 1991, Nucl. Phys. A, 535, 331

Lentz, E. J., Mezzacappa, A., Bronson Messer, O. E., Hix, W. R., \& Bruenn, S. W. 2012a, ArXiv e-prints, 1206.1086

Lentz, E. J., Mezzacappa, A., Bronson Messer, O. E.,

Liebendörfer, M., Hix, W. R., \& Bruenn, S. W. 2012b, ApJ, 747,73

Liebendörfer, M. 2005, ApJ, 633, 1042

Liebendörfer, M., Messer, O. E. B., Mezzacappa, A., Bruenn, S. W., Cardall, C. Y., \& Thielemann, F.-K. 2004, ApJS, 150, 263

Liebendörfer, M., Mezzacappa, A., \& Thielemann, F.-K. 2001, Phys. Rev. D, 63, 104003:1

Liebendörfer, M., Rampp, M., Janka, H.-T., \& Mezzacappa, A. 2005, ApJ, 620, 840

Liebendörfer, M., Whitehouse, S. C., \& Fischer, T. 2009, ApJ, 698,1174

Livne, E., Burrows, A., Walder, R., Lichtenstadt, I., \& Thompson, T. A. 2004, ApJ, 609, 277

Livne, E., Dessart, L., Burrows, A., \& Meakin, C. A. 2007, ApJS, 170,187

Marek, A., Dimmelmeier, H., Janka, H.-T., Müller, E., \& Buras, R. 2006, A\&A, 445, 273

Marek, A., \& Janka, H. 2009, ApJ, 694, 664

Mezzacappa, A., \& Bruenn, S. W. 1993, ApJ, 405, 669

Mignone, A., \& Bodo, G. 2005, MNRAS, 364, 126

Müller, B., Dimmelmeier, H., \& Müller, E. 2008, A\&A, 489, 301

Müller, B., Janka, H., \& Dimmelmeier, H. 2010, ApJS, 189, 104

Müller, E., \& Janka, H.-T. 1997, A\&A, 317, 140

Murphy, J. W., \& Burrows, A. 2008, ApJ, 688, 1159

Nordhaus, J., Burrows, A., Almgren, A., \& Bell, J. 2010, ApJ, 720,694

Ohnishi, N., Kotake, K., \& Yamada, S. 2006, ApJ, 641, 1018

Ott, C. D., Dimmelmeier, H., Marek, A., Janka, H.-T., Hawke, I., Zink, B., \& Schnetter, E. 2007a, Phys. Rev. Lett., 98, 261101:1

Ott, C. D., Dimmelmeier, H., Marek, A., Janka, H.-T., Zink, B., Hawke, I., \& Schnetter, E. 2007b, Class. Quantum Grav., 24, S139

Ott, C. D., et al. 2011, Physical Review Letters, 106, 161103

Pejcha, O., \& Thompson, T. A. 2012, ApJ, 746, 106

Pons, J. A., Miralles, J. A., \& Ibanez, J. M. A. 1998, A\&AS, 129, 343

Pons, J. A., Reddy, S., Prakash, M., Lattimer, J. M., \& Miralles, J. A. 1999, ApJ, 513, 780

Pruet, J., Hoffman, R. D., Woosley, S. E., Janka, H.-T., \& Buras, R. 2006, ApJ, 644, 1028

Pruet, J., Woosley, S. E., Buras, R., Janka, H.-T., \& Hoffman,

R. D. 2005, ApJ, 623, 325

Quirk, J. J. 1994, Int. J. Num. Meth. in Fluids, 18, 555

Raffelt, G. G. 2001, ApJ, 561, 890

Rampp, M., \& Janka, H.-T. 2002, A\&A, 396, 361

Rantsiou, E., Burrows, A., Nordhaus, J., \& Almgren, A. 2011, ApJ, 732, 57

Reddy, S., Prakash, M., Lattimer, J. M., \& Pons, J. A. 1999,

Phys. Rev. C, 59, 2888

Sagert, I., Fischer, T., Hempel, M., Pagliara, G.,

Schaffner-Bielich, J., Mezzacappa, A., Thielemann, F.-K., \&

Liebendörfer, M. 2009, Physical Review Letters, 102, 081101:1

Scheck, L. 2006, PhD thesis, Technische Universtiät München

Scheck, L., Janka, H.-T., Foglizzo, T., \& Kifonidis, K. 2008, A\&A, 477, 931

Scheidegger, S., Fischer, T., Whitehouse, S. C., \& Liebendörfer, M. 2008, A\&A, 490, 231

Shen, H., Toki, H., Oyamatsu, K., \& Sumiyoshi, K. 1998, Nucl. Phys. A, 637, 435

Schinder, P. J. 1990, ApJS, 74, 249

Shlomo, S., Kolomietz, V. M., \& Colò, G. 2006, European Physical Journal A, 30, 23

Steiner, A. W., Lattimer, J. M., \& Brown, E. F. 2010, ApJ, 722, 33

Suwa, Y., Kotake, K., Takiwaki, T., Whitehouse, S. C., Liebendörfer, M., \& Sato, K. 2010, PASJ, 62, L49+ Suzuki, H. 1990, PhD thesis, , University of Tokyo, (1990)

Takiwaki, T., Kotake, K., \& Suwa, Y. 2012, ApJ, 749, 98

Thompson, C. 2000, ApJ, 534, 915

Thompson, T. A., Quataert, E., \& Burrows, A. 2005, ApJ, 620, 861

Wanajo, S., Janka, H.-T., \& Müller, B. 2011, ApJ, 726, L15 
Wongwathanarat, A., Janka, H., \& Müller, E. 2010, ApJ, 725, L106

Woosley, S. E., Heger, A., \& Weaver, T. A. 2002, Rev. Mod. Phys., 74, 1015

Woosley, S. E., \& Weaver, T. A. 1995, ApJS, 101, 181
Yamada, S., Janka, H.-T., \& Suzuki, H. 1999, A\&A, 344, 533

Yamasaki, T., \& Yamada, S. 2007, ApJ, 656, 1019 